\title{
QS能
}

Joaquín María Núñez Varo*

María del Mar Gómez González**

\section{EL IMPACTO A CORTO Y MEDIO PLAZO DEL APOYO DE ICEX}

La estrategia de evaluación, puesta en marcha por ICEX a partir de 2011, contemplaba llegar a analizar el efecto de su apoyo en las principales variables económicas de las empresas como base para la toma de decisiones y la acción orientada a resultados. Una exhaustiva y compleja evaluación, cuyo diseño se inició en 2017 y que en este artículo se detalla, nos ha permitido alcanzar una estimación de ese impacto, unos resultados que refuerzan y consolidan lo apuntado en las evaluaciones de instrumentos y programas de ICEX realizadas internamente. Los resultados positivos muestran un impacto diferente por perfiles de empresas, y el análisis de la evolución temporal permite observar que el fuerte impulso de la exportación de bienes que se produce de forma inmediata gracias al apoyo de ICEX no se mantiene a medio plazo para todos los tipos de empresa.

Palabras clave: evaluación de impacto, evaluación de políticas públicas, internacionalización empresarial, métodos cuantitativos.

Clasificación JEL: C02, C44, C52, C93, L53.

\section{Introducción}

Dentro del Plan de Acción para la Internacionalización de la Empresa Española 20172018 se contemplaba realizar una evaluación de impacto del apoyo prestado por la Secretaría de Estado de Comercio a la empresa a través de todos sus instrumentos de apoyo. En el marco del convenio de colaboración entre el

*Analista del Departamento de Evaluación de ICEX España Exportación e Inversiones, Profesor Asociado de la Universidad Carlos III de Madrid y Miembro del Comité de Asesoramiento Científico del Centro de Investigación en Economía Digital de la Universidad Autónoma de Madrid.

**Directora Adjunta de Sostenibilidad y Evaluación de ICEX España Exportación e Inversiones y Profesora Colaboradora en el Máster de Comercio Exterior de la Universidad Carlos III de Madrid. Versión de noviembre de 2020.

DOI: https:/doi.org/10.32796/bice.2020.3130.7142
Banco de España (BdE), el ICEX, el Instituto Nacional de Estadística (INE) y la Secretaría de Estado de Comercio, para la evaluación de dicho plan, se incluyó la realización de una evaluación de impacto del apoyo de ICEX.

Ha habido una estrecha colaboración con el equipo de esta secretaría, de la que ICEX depende, para diseñar la mejor evaluación posible en función de los datos disponibles, de forma que se aprovechara todo el trabajo previo realizado en el Departamento de Evaluación de ICEX, que puede verse en Núñez (2020). En este artículo, el primero de una serie que pensamos publicar, mostramos un resumen del trabajo realizado, que podrá consultarse en breve y de forma íntegra en la web de ICEX. $\square$ 
Las preguntas que hemos planteado en esta evaluación son las siguientes:

¿Hay algún efecto observable en las magnitudes económicas entre aquellas empresas que han recibido apoyo de ICEX?

¿Cuáles de esos efectos son atribuibles al apoyo de ICEX y en qué medida?

¿Hay diferencias en los efectos observables y atribuibles entre los diferentes tipos de empresa existentes?

¿Qué retorno de inversión vía impuestos es atribuible al apoyo de ICEX?

Para dar una respuesta aproximada a la pregunta del retorno de inversión pública hemos realizado un sencillo cálculo sobre el retorno vía impuestos del efecto esperado atribuible al apoyo de ICEX. A falta de poder disponer para este trabajo de datos desagregados y completos sobre las cuentas fiscales tanto de las empresas como de los empleados, este cálculo se ha realizado de forma muy simplificada, y sus resultados deben tomarse como una mera aproximación cuyo ánimo está bien lejos de pretender suplir el necesario análisis con microdatos sobre fiscalidad de empresas fusionados con el resto de microdatos que hemos podido emplear en la evaluación.

\section{Metodología}

Aunque hemos realizado varios ejercicios de evaluación complementarios sobre cuatro conjuntos de datos distintos que abarcan periodos de tiempo diferentes, en este artículo presentamos solo la parte correspondiente al realizado sobre la base de datos construida combinando información del Directorio Central de Empresas (DIRCE), datos de comercio de bienes de Aduanas y datos de acceso a programas de asistencia o apoyo de ICEX entre los años 2014 y 2018, en virtud del convenio mencionado anteriormente. Esta base, que llamamos internamente Base_INE, es idéntica a la denominada Caracterización de la Empresa Exportadora y con Potencial Exportador (CEEPE) en el informe de evaluación del Plan de Acción para la Internacionalización de la Economía Española 2017-2018, elaborado por la Secretaría de Estado de Comercio en 2020.

En todos los análisis hemos planteado una perspectiva de permanencia en términos económicos del resultado obtenido, de forma que no solo se constate si el apoyo brinda un efecto rápido en el tiempo que ayude al impulso internacional de una empresa, sino si este efecto se mantiene, para asegurar que la empresa potencie y consolide su actividad exterior. Hemos determinado como plazo razonable para comprobar este efecto mantenido cuatro años después del inicio del apoyo. Esta decisión se apoya en tres hechos empíricos:

- El primer bache para una empresa que sale al mercado exterior son los tres años iniciales, periodo tras el que la empresa puede dejar el mercado internacional o mejorar su estrategia y aumentar su presencia exterior.

- Las empresas, en términos generales, no suelen utilizar los apoyos de ICEX más de tres años seguidos (Tabla 1). La mayoría de las empresas ha utilizado servicios de apoyo solo un año, y en caso de utilizarlos más tiempo, el número esperado de años no llega a dos. Las que nos utilizan más de tres años suelen seguir secuencias de participación en instrumentos de apoyo a exhibición (participación en ferias, fundamentalmente) o de capacitación (asistencia a jornadas o seminarios). 
TABLA 1

AÑOS DE UTILIZACIÓN DE SERVICIOS DE ICEX EN EL PERIODO 2014-2018

\begin{tabular}{|l|c|c|c|c|}
\hline \multicolumn{1}{|c|}{ Periodo } & Media & $\begin{array}{c}\text { Percentil } \\
\mathbf{9 0}\end{array}$ & $\begin{array}{c}\text { Máximo } \\
\text { Intervalo de confianza para la } \\
\text { media con una confianza del 95\% }\end{array}$ \\
\hline $\begin{array}{l}\text { Servicios de apoyo o de información para todas las empresas } \\
\begin{array}{l}\text { Servicios de apoyo o de información para empresas que han } \\
\text { utilizado algún servicio de apoyo durante el periodo }\end{array}\end{array}$ & $\begin{array}{l}1,68 \\
\text { Servicios de apoyo de ICEX durante el periodo }\end{array}$ & 3 & 5 & 5 \\
\hline Fuente: Departamento de Evaluación de ICEX. & 1,82 & 4 & 5 & {$[2.404,2.437]$} \\
\hline
\end{tabular}

- Nuestras evaluaciones de impacto internas muestran de forma consistente que el efecto en entrada en mercados se produce uno o dos años después del apoyo prestado por ICEX.

Para estimar el impacto del apoyo de ICEX, hemos contemplado una ventana máxima de cuatro años a partir del inicio del apoyo, lo que ofrece un marco temporal máximo de cinco años completos. En términos del retorno de la inversión pública vía impuestos, esto implica que todo el presupuesto de los cinco años se dedica exclusivamente al apoyo a las empresas que lo han iniciado en ese periodo con el fin de que mejoren su competitividad y, por ende, sus cifras económicas (cifra de negocios, exportación de bienes y empleo). Como esta ventana de tiempo es móvil, si no ha habido cambio ni en la estructura empresarial española ni en el tipo de cliente que recurre al ICEX ni en la política de la propia institución, cabe esperar un efecto similar de forma independiente al año en que fijemos el inicio de esa ventana.

Nuestro interés, como en todo ejercicio de evaluación, es responder a la pregunta de si podemos rechazar la hipótesis de que no hay una relación entre el apoyo y el efecto observado y, en su caso, ofrecer una estimación del incremento en la magnitud del resultado que cabe atribuir al apoyo de ICEX. Esto se consigue mediante el diseño de un experimento, y no debe confundirse con la modelización de la realidad teniendo en cuenta todos los factores y la interacción entre estos. Este ejercicio debe ser posterior y fundarse en los hallazgos de esta evaluación.

\section{Lógica de la intervención}

El Gráfico 1 representa la lógica de la intervención reconstruida para el apoyo a la internacionalización que ofrece ICEX.

De acuerdo con la lógica planteada, el proceso de evolución de una empresa hasta llegar a ser potencial candidato de apoyo de ICEX para impulsar su proceso de internacionalización requiere que esta disponga de un producto o servicio propio y haya adquirido un cierto músculo financiero para crecer en los mercados internacionales. Por lo tanto, un riesgo que debemos contemplar en el análisis es que una falta de solvencia inicial de la empresa o sobrevenida interrumpa su aventura internacional. Asimismo, y tras el apoyo de ICEX, es esperable un incremento en sus cifras de exportación de bienes o servicios, y la buena marcha de la empresa puede derivar en establecimientos productivos en mercados exteriores o en ser adquirida por otro grupo empresarial. En estos dos últimos casos, la evolución sería esperable y positiva, pero al tener como unidad muestral un constructo contable (CIF) y no líneas de $\triangleright$ 


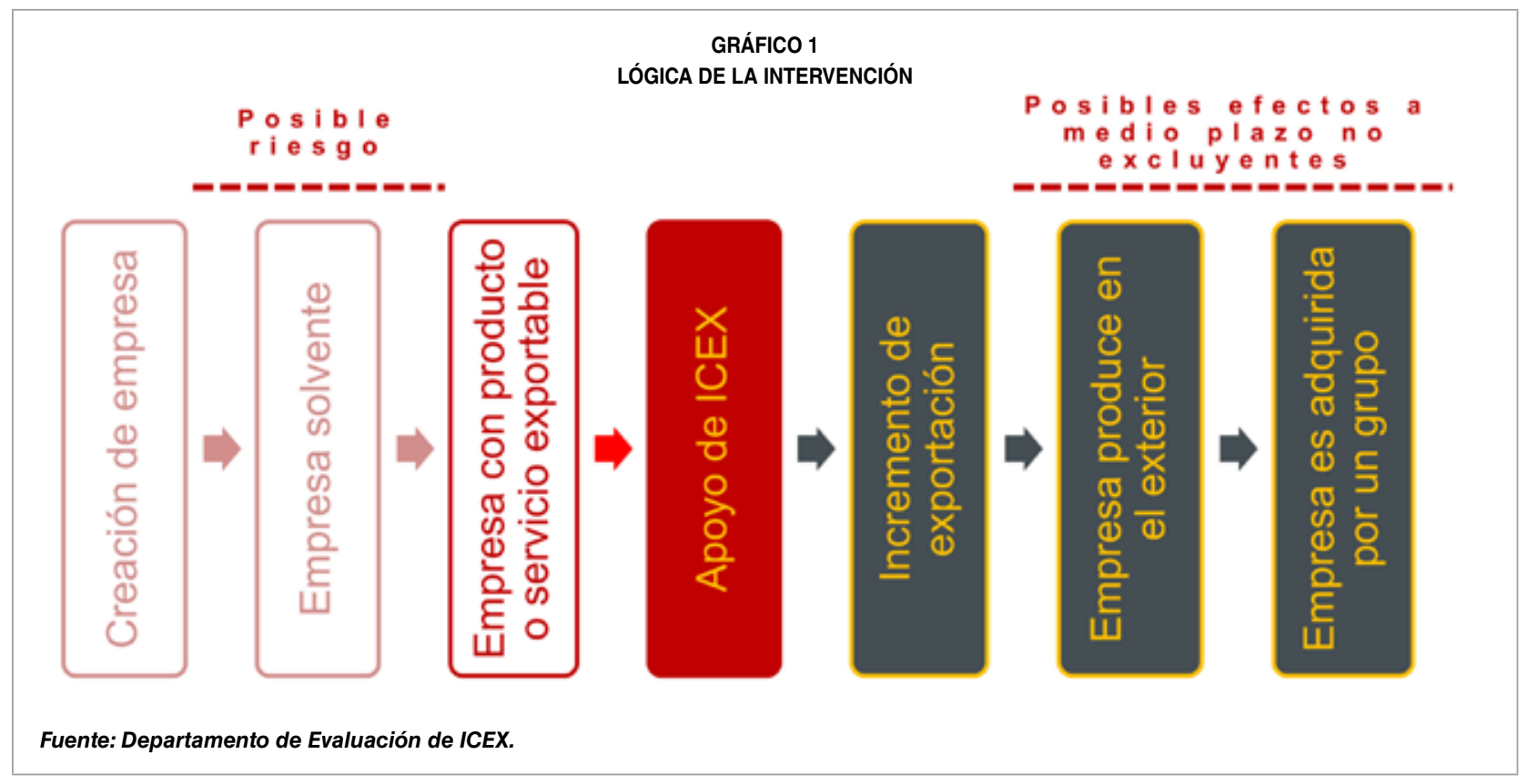

producción (que se mantienen a pesar de cambiar de manos empresariales), el análisis reflejaría un descenso en las cifras de exportación, lo que haría pensar en un empeoramiento en la evolución de esta variable, si bien en realidad ese descenso ocultaría efectos positivos que se reflejarían en otras variables, además de dotar a la empresa de una mayor competitividad y resiliencia frente a coyunturas adversas. Ante el problema de no disponer de variables adicionales que reflejen estas circunstancias, se ha procedido a clusterizar la base del DIRCE, lo que ha permitido realizar una evaluación por separado para cada tipo de empresa, las cuales representan diferentes perfiles y permiten interpretar de forma conjunta la evolución de todas las variables a la vez y valorar si un descenso en exportación de bienes en un contexto de aumento de cifra de negocios y de productividad, por ejemplo, no implica necesariamente un empeoramiento en los resultados empresariales, sino quizá la consecuencia de una implantación productiva en el exterior, de forma que se sigue mejorando la facturación, pero se reduce la exportación desde España, lo que en ciertos sectores y mercados es necesario para evitar, por ejemplo, barreras no arancelarias que el mercado de destino impone ${ }^{1}$.

\section{Diseño de la evaluación}

Hemos seguido los siguientes pasos para determinar la mejor manera de llevar a cabo esta evaluación.

\section{Paso 1. Definir de forma conveniente la variable de tratamiento}

Este paso implica poder medir con precisión si una empresa ha recibido algún apoyo $\triangleright$

\footnotetext{
1 Hay evidencia empírica de que las empresas más productivas eligen atender algunos mercados exteriores mediante la implantación productiva (lo que requiere inversión directa), en función de los costes de entrada o de la fortaleza de la competencia local, si bien la heterogeneidad en las características de estas firmas es un elemento esencial para poder aislar bien este hecho. Ver, por ejemplo, Helpman (2003) o Yang-Ming (2009). También existe evidencia empírica de que la denominada inversión directa horizontal (fusiones con entidades locales) crece relativamente más rápido que las exportaciones en las industrias de bienes diferenciados cuando los costes de transacción caen. Ver, por ejemplo, Muendler (2014).
} 
para su proceso de internacionalización o no. Se convino con la Secretaría de Estado de Comercio la siguiente clasificación de los servicios que ofrece ICEX a la empresa:

- Apoyo. En esta categoría se incluyen los instrumentos de apoyo a la promoción exterior (que suele prestar ICEX con apoyo de la red de Oficinas Económicas y Comerciales de España en el exterior), los programas PIPE-Next y Cre100do, las diferentes jornadas y seminarios, los servicios personalizados (también prestados principalmente por la red) y los servicios de asesoramiento personalizado.

- Información. En esta categoría se incluyen el servicio de Oportunidades de $\mathrm{Ne}$ gocio, las consultas atendidas por cualquier nivel de Ventana Global, los seminarios colgados para su visualización en el Aula Virtual y el servicio de Costes de Establecimiento.

Por otra parte, se convino también en poder estudiar de forma separada las empresas que habían recibido apoyo por primera vez en el periodo y las que ya lo habían recibido con anterioridad. Para ello se definió una variable denominada «veteranía», que identifica a aquellas empresas que han utilizado algún instrumento de apoyo de ICEX entre los años 2003 y 2013.

\section{Paso 2. Determinar el marco temporal más adecuado en el que observar efectos tras la prestación del apoyo}

Hemos determinado el primer año del periodo 2014-2018 en el que cada empresa recibe un servicio de apoyo y, a partir de este valor, el desfase entre el inicio del apoyo y el año en que se mide la variable de interés. Aunque nos planteábamos realizar estimaciones diferenciadas según el año de inicio del apoyo, nos encontramos con la dificultad de que no era fácil distinguir grupos de control separados para cada uno de los años. Por ello, solo hemos considerado el año de inicio del apoyo para poder obtener evidencia de cómo evoluciona el efecto observado tras el primer apoyo, y en la estimación del impacto decidimos no hacer dicha distinción y conformarnos con contemplar el efecto agregado a cuatro años vista, tomando como situación inicial de las empresas su estado en 2014 y como situación final su estado en 2018, último año del periodo. Esta definición implica una cierta dispersión en el efecto atribuido al apoyo de ICEX, pues estamos mezclando efectos observados en empresas similares en años diferentes tras el inicio del apoyo, y a esta dispersión se añade la debida a que tomamos como apoyo servicios que tienen objetivos diferentes e intensidades de apoyo distintas. Si bien esto constituye un riesgo nada desdeñable para la validez interna del experimento, nos permitía poder realizar la estimación del impacto en grupos similares de empresas, y tener una primera aproximación del efecto atribuible al apoyo de ICEX, con independencia de la necesidad de cada empresa y de cuándo dicha empresa haya recurrido a ICEX.

Paso 3. Determinar de forma conveniente el grupo de control más adecuado

La determinación del grupo de control se debe hacer partiendo del conocimiento profundo del propio grupo de tratamiento. Aunque propusimos incluir las variables de Informa que permiten observar la evolución en materia de competitividad de las empresas que $\triangleright$ 
han sido alguna vez clientes de ICEX, o sus equivalentes en el DIRCE, lo que habría permitido segmentar toda la población en grupos predefinidos que han surgido del análisis realizado hasta la fecha por el Departamento de Evaluación y afinar estos grupos considerando toda la población de empresas incluida en el DIRCE, finalmente solo pudimos contar con las siguientes variables de caracterización de la empresa:

- Comunidad autónoma donde se sitúa la sede de la empresa.

- Código CNAE principal de la empresa.

- Edad de la empresa.

- Número de trabajadores.

- Cifra de negocios.

- Exportación de bienes.

A partir de estas variables hemos definido dos nuevas variables secundarias:

- Intensidad exportadora: exportación de bienes / cifra de negocios.

- Productividad: cifra de negocio / número de trabajadores.

Con las variables disponibles era difícil decidir qué empresas no podían ser objeto de apoyo de ICEX, pues trabajamos con empresas de todo tipo, y ni siquiera el hecho de que mostraran algún valor en la cifra de exportación de bienes nos era de utilidad, pues muchos de nuestros apoyos van dirigidos a empresas que exportan servicios, y tenemos también instrumentos de apoyo destinados a que las empresas se inicien en el mercado internacional. Esto implica la necesidad de comparar empresas que han recibido apoyo con sus pares, lo que solo puede hacerse mediante alguna técnica de emparejamiento, pero descartamos la técnica de propensity score matching por dos motivos fundamentales, uno técnico y otro pragmático:

El motivo técnico consiste en que para utilizar la técnica de propensity score matching deben conocerse con exactitud los criterios por los que una empresa es aceptada para recibir el apoyo, y debemos tener, tanto para las empresas de tratamiento como para las de control, las variables que informen del cumplimiento de estos criterios. En nuestro caso, salvo para algunos apoyos muy concretos (como ICEX-Next o el apoyo dentro de planes sectoriales), los criterios aplicables son amplios y flexibles, de forma que ninguna de las variables de la base construida era suficiente para determinar su cumplimiento.

El motivo pragmático consiste en que el propensity score matching es en el fondo una caja negra, y su falta de transparencia nos impedía extraer conclusiones válidas por tipo de empresa.

Optamos por utilizar la técnica de emparejamiento exacto forzado (coarsened exact matching, CEM), combinada con una técnica de diferencias en diferencias mediante una estimación por datos de panel, lo que, a nuestro juicio, permite maximizar la validez interna del experimento, pues en las características diferenciales de cada tipo de empresa identificado se encuentran reflejados de forma implícita muchos de los factores que pueden incidir en los efectos observados.

\section{Adecuación de las variables}

Se ha realizado una transformación logarítmica de las variables continuas disponibles, y hemos comprobado que esta transformación sigue una distribución que se aproxima a $\triangleright$ 
una mezcla de normales en todas las subpoblaciones definidas por el tipo de apoyo y el estado de veteranía en el periodo. Esta circunstancia aconseja acometer la búsqueda de estas diferentes subpoblaciones mediante técnicas de reducción de la dimensión. Se ha utilizado una técnica de clusterización que ha encontrado grupos más homogéneos donde estas variables siguen distribuciones próximas a la normal, lo que reduce dificultades en el análisis posterior, en particular problemas de validez interna y estadística en el experimento $\mathrm{y}$ de sesgo en las estimaciones.

\section{Segmentación de empresas}

Hemos sometido toda la base, constituida por 1.555.987 empresas, a un algoritmo de clusterización por K-medias, idóneo para descubrir agrupaciones de observaciones (en nuestro caso, empresas) en las que la distribución conjunta de las variables explicativas dentro de cada clúster siga una distribución normal multidimensional. Los resultados combinados de tres criterios de calidad de clusterización distintos - criterio de la Pseudo-T, criterio de la Pseudo-F y criterio cúbico de clusterizaciónhan sugerido la existencia de siete clústeres en la muestra, que en lo sucesivo llamaremos simplemente tipos de empresa, cuyas características y descripciones se muestran en la Tabla 2.

Los Gráficos 2 a 5 muestran de forma comparada la evolución de las características entre empresas receptoras de apoyo de ICEX (apoyo), servicios de información (otro) y grupo de empresas sin apoyo (control) hasta el año 2018, en escala logarítmica para ofrecer los datos con mayor claridad.

TABLA 2

CARACTERIZACIÓN DE LOS TIPOS DE EMPRESAS SURGIDOS MEDIANTE EL CLUSTERIZADO DE K-MEDIAS

\begin{tabular}{|c|c|c|c|c|c|c|}
\hline Clúster & Descripción & $\begin{array}{l}\text { N. }{ }^{\circ} \text { de } \\
\text { empresas }\end{array}$ & $\begin{array}{l}\text { Edad } \\
\text { (años) }\end{array}$ & $\begin{array}{l}\mathrm{N} .^{\circ} \text { de } \\
\text { trabajadores }\end{array}$ & $\begin{array}{l}\text { Productividad } \\
\text { (euros/trabajador) }\end{array}$ & $\begin{array}{l}\text { Exportación de } \\
\text { bienes (euros) }\end{array}$ \\
\hline TODAS & Todas las empresas del DIRCE & 1.555 .987 & 9,51 & 6,46 & $358.155,49$ & $1.059,38$ \\
\hline 1 & $\begin{array}{l}\text { Microempresas productivas de más de } 10 \\
\text { años de vida y con tímida presencia en el } \\
\text { exterior }\end{array}$ & 376.958 & 12,48 & 2,82 & $82.790,53$ & $2.552,99$ \\
\hline 2 & $\begin{array}{l}\text { Microempresas poco productivas de } \\
\text { menos de } 10 \text { años de vida y tímida } \\
\text { presencia en el exterior }\end{array}$ & 91.760 & 7,66 & 2,48 & 0,39 & $1.215,79$ \\
\hline 3 & $\begin{array}{l}\text { Empresas medianas y grandes de más de } \\
15 \text { años de vida muy productivas y } \\
\text { volcadas en el exterior }\end{array}$ & 124.389 & 15,41 & 57,17 & $179.671,33$ & $1.521 .868,25$ \\
\hline 4 & $\begin{array}{l}\text { Microempresas de reciente creación muy } \\
\text { productivas y volcadas en el exterior }\end{array}$ & 314.323 & 1,17 & 0,83 & $63.416,94$ & $11.160,66$ \\
\hline 5 & $\begin{array}{l}\text { Microempresas de más de } 10 \text { años de } \\
\text { vida muy productivas y muy volcadas en } \\
\text { el exterior }\end{array}$ & 520.954 & 11,11 & 0,31 & $926.718,42$ & $40.524,98$ \\
\hline 6 & $\begin{array}{l}\text { Pequeñas empresas de más de } 12 \text { años } \\
\text { de vida volcadas en el exterior }\end{array}$ & 24.632 & 14,60 & 20,68 & 0,00 & $11.756,01$ \\
\hline 7 & $\begin{array}{l}\text { Microempresas de menos de } 10 \text { años de } \\
\text { vida con baja productividad y tímida } \\
\text { presencia en el exterior }\end{array}$ & 102.971 & 9,25 & 6,94 & $9.871,54$ & $1.211,89$ \\
\hline \multicolumn{7}{|c|}{$\begin{array}{l}\text { Clusterización por K-medias de las empresas del DIRCE por sus características de } 2014 \text {. } \\
\text { R-cuadrado general esperado = 0,88482. }\end{array}$} \\
\hline \multicolumn{7}{|c|}{ Fuente: CEEPE y elaboración propia. } \\
\hline
\end{tabular}




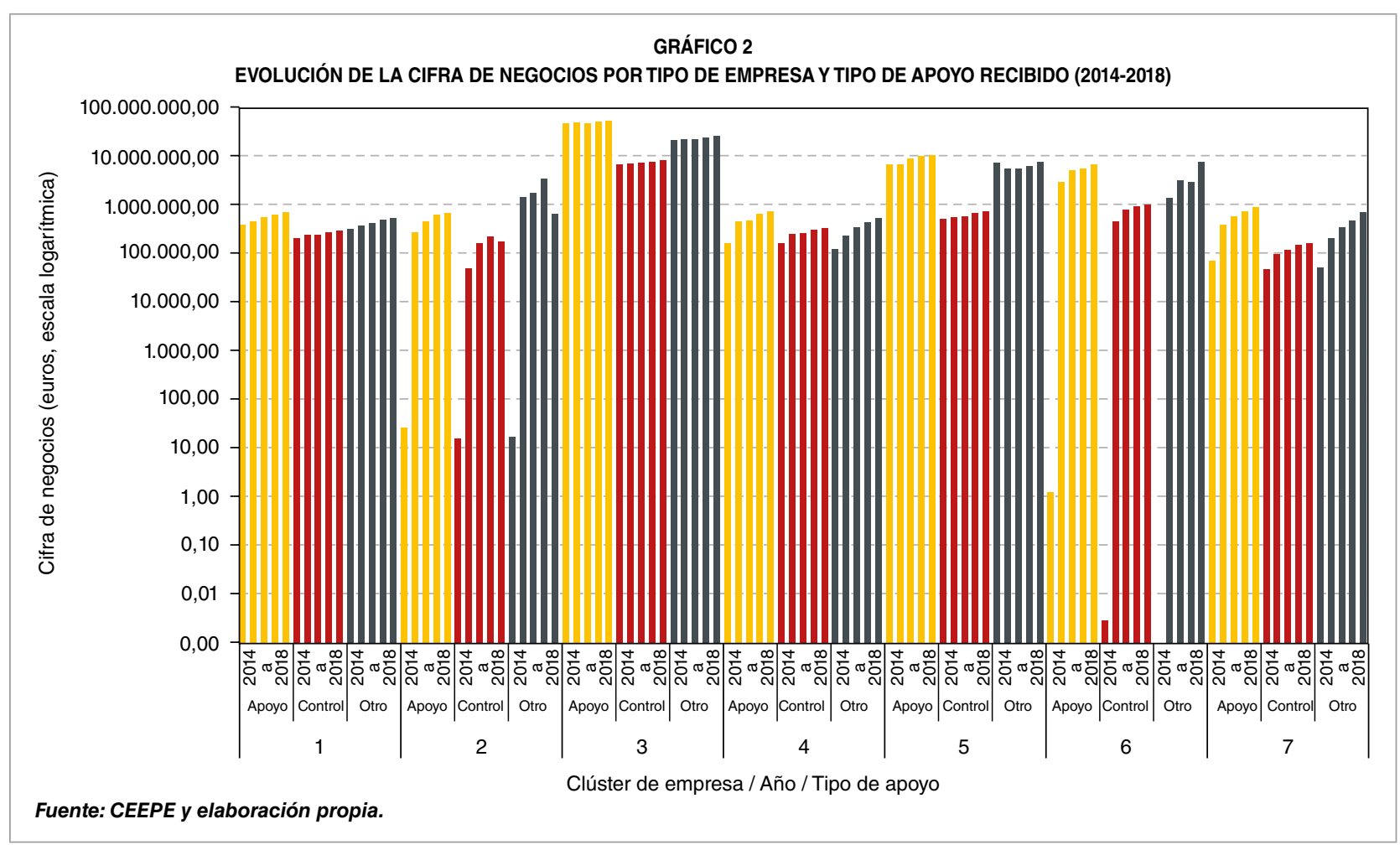

En términos de cifra de negocios, salvo el clúster 2 (microempresas poco productivas de menos de diez años de vida y tímida presencia en el exterior), las empresas que han recibido apoyo de
ICEX tienen cifras de negocio considerablemente mayores que las que no lo han recibido. Además, estas cifras, bien se mantienen a lo largo del periodo, bien van creciendo de forma paulatina.

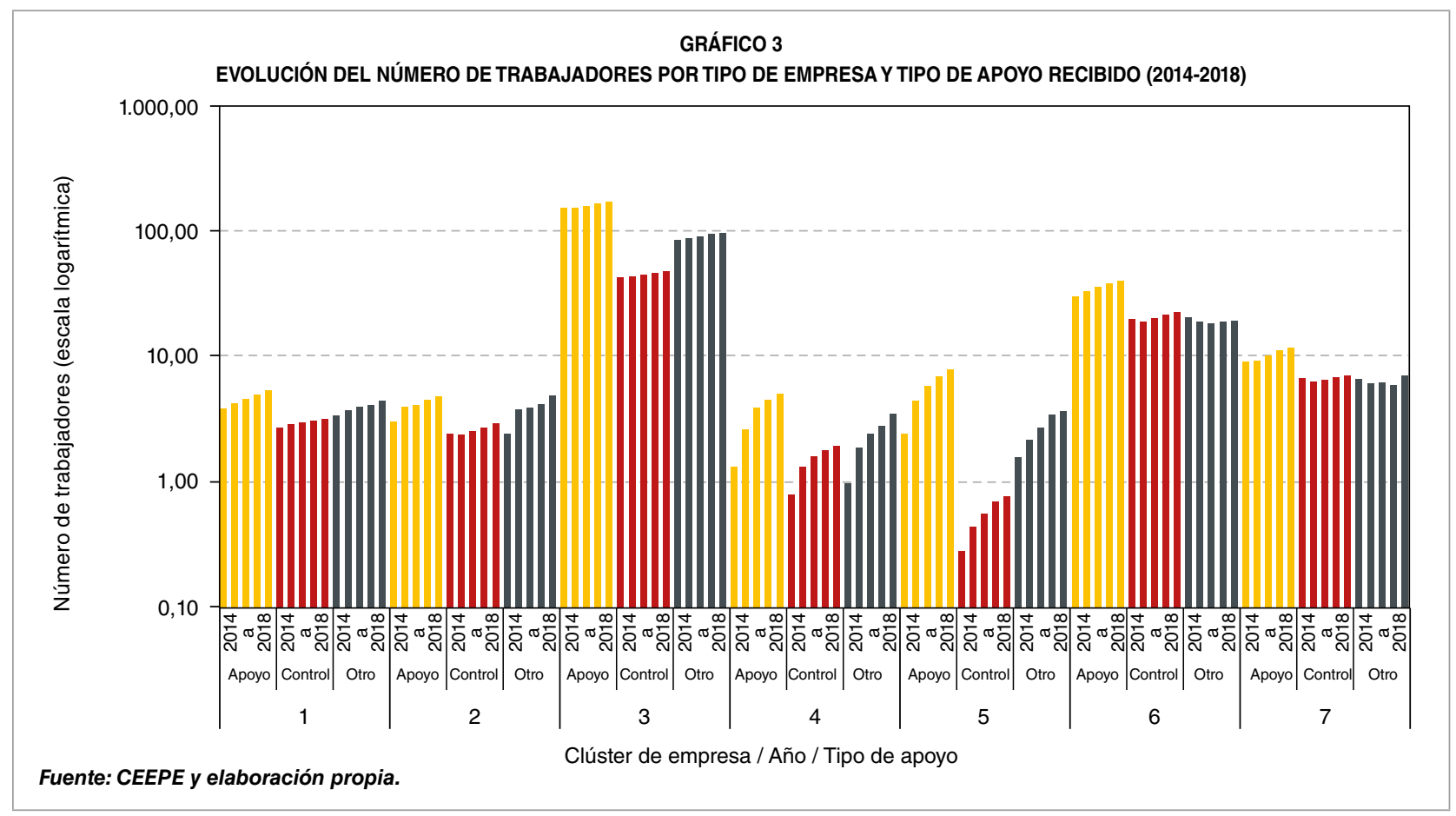




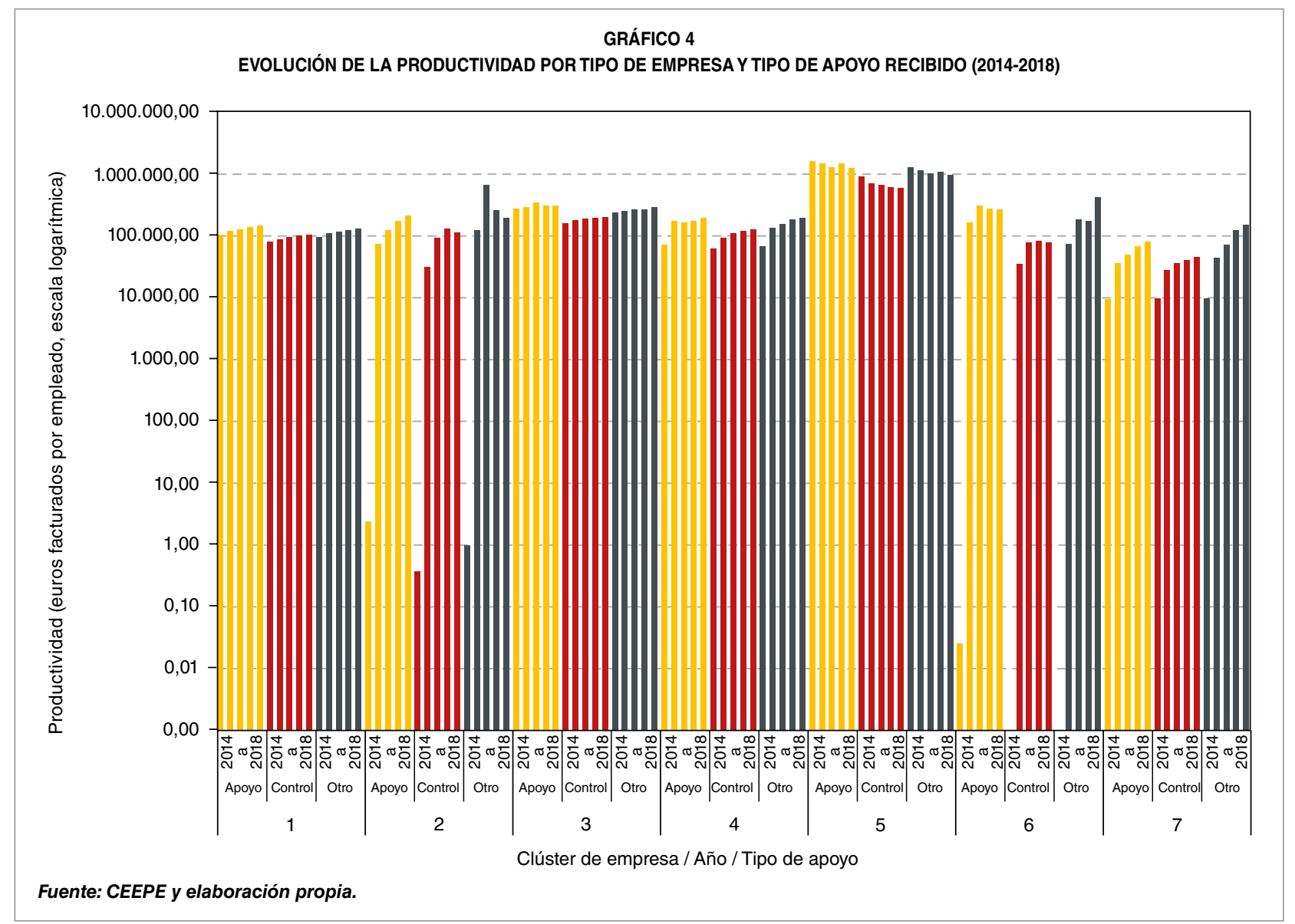

En términos de plantilla, las empresas que han recibido apoyo de ICEX tienen un número de empleados bastante superior que las que no lo han recibido, y este número va incrementándose de forma paulatina.

En términos de productividad, las empresas que han recibido apoyo de ICEX tienen en general una mayor productividad que las que no lo han recibido, y se incrementa poco a poco, salvo en los clústeres 5 y 6 , correspondientes a microempresas de más de diez años de vida muy productivas y muy volcadas en el exterior, y a pequeñas empresas de más de doce años de vida volcadas en el exterior, en las cuales esta productividad se estanca o retrocede ligeramente.

En términos de exportación de bienes, las empresas que han recibido apoyo de ICEX tienen, en general, unas cifras considerablemente mayores que las que no lo han recibido, y estas se incrementan con el discurrir de los años, salvo en las empresas del clúster 2 (microempresas poco productivas de menos de diez años de vida y tímida presencia en el exterior), donde el último año disminuyen. Es de destacar también que las empresas de este clúster que han solicitado servicios de información tienen mejores cifras de exportación que las que han recibido servicios de apoyo. Es posible que estas empresas reciban apoyos de otros organismos y tan solo requieren información puntual por parte de ICEX, o que no conozcan la amplia cartera de servicios de apoyo que ICEX puede poner a su disposición.

Este análisis es meramente descriptivo y muestra tan solo la evolución de cada tipo $\square$ 


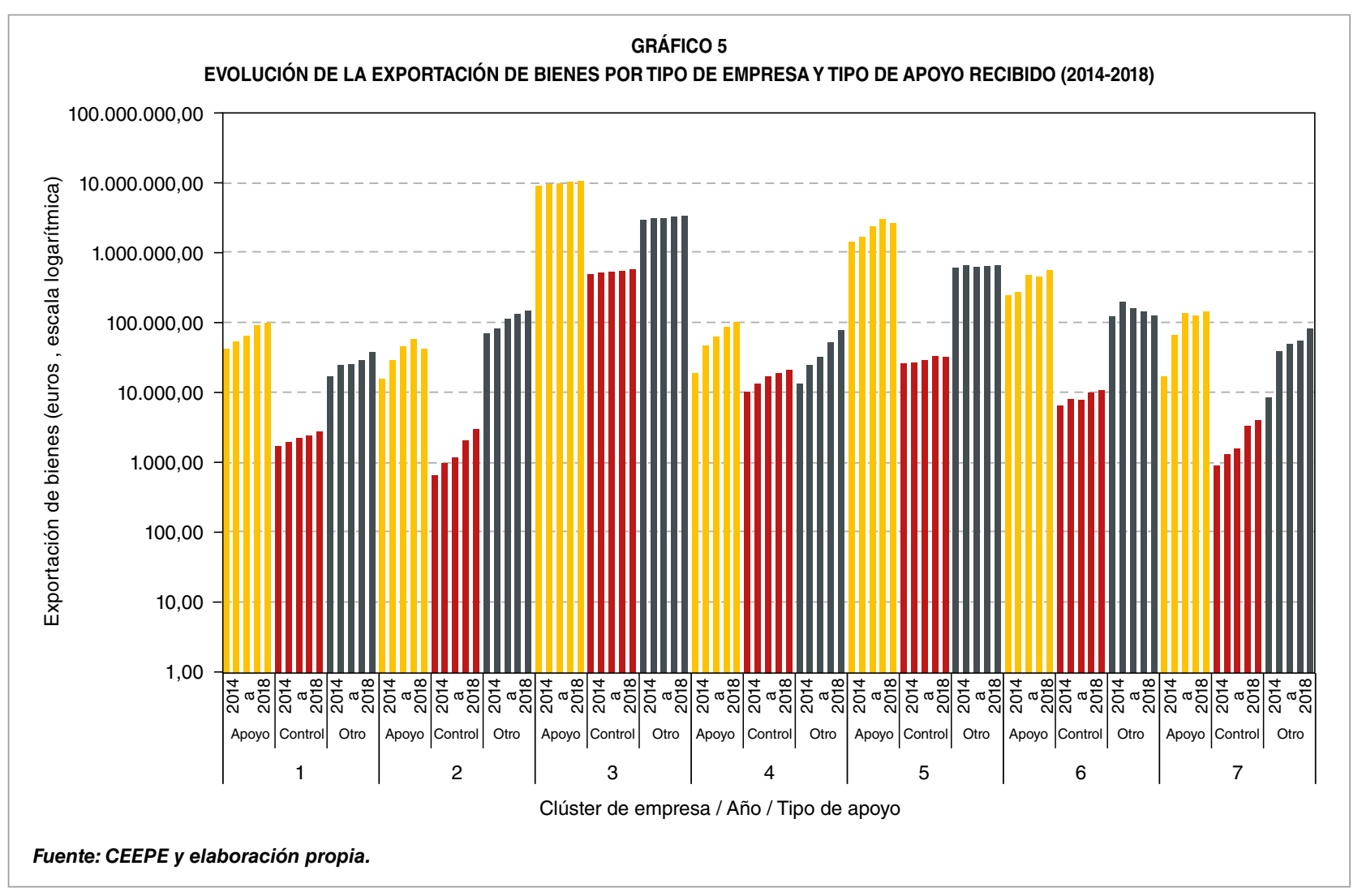

de empresa, pero no nos dice nada de cómo el apoyo ha podido incidir en la evolución de estas magnitudes.

\section{Análisis de contribución del apoyo de ICEX}

Para dar contestación a la pregunta de si puede atribuirse una relación de causa-efecto entre el apoyo de ICEX y el efecto observado, $y$ en caso afirmativo, estimar en qué medida el apoyo de ICEX es responsable de este efecto, hemos procedido a combinar dos técnicas de estimación: el emparejamiento exacto forzado (CEM) y la técnica de diferencias en diferencias mediante una estimación por datos de panel. En particular, hemos estimado diferentes ecuaciones para las distintas variables de interés, cuya especificación figura a continuación.

Ecuación para la variable exportación de bienes:

$$
\begin{aligned}
& \ln \left(1+\text { exp_tot }_{u v i l}\right)=\alpha+\alpha_{u}+\alpha_{u v}+\alpha_{u v i}+\beta_{1 u v} * t+\beta_{2 u v} * D_{i}+\beta_{3 u v} \\
& * D_{i} * t+\beta_{4 u v} * \ln \left(1+e^{2} d_{a d} d_{u v i t}\right)+\beta_{5 u v} * \ln \left(1+\text { trabele }_{u v i t}\right)+ \\
& \beta_{6 u v} * \ln \left(1+\text { productiv }{ }_{u v i t}\right)+\beta_{7 u v} * \ln \left(1+\text { intens_exp }{ }_{u v i t}\right)+\varepsilon_{u v i t}
\end{aligned}
$$

Ecuación para la variable cifra de negocios:

$\ln \left(1+c n_{u v i t}\right)=\alpha+\alpha_{u}+\alpha_{u v}+\alpha_{u v i}+\beta_{1 u v} * t+\beta_{2 u v} * D_{i}+\beta_{3 u v} \star D_{i} \star t$

$+\beta_{4 u v} * \ln \left(1+e d a d_{u v i t}\right)+\beta_{5 u v} * \ln \left(1+e x p \_t o t_{u v i t}\right)+$

$\beta_{6 u v}{ }^{*} \ln \left(1+\right.$ productiv $\left._{u v i t}\right)+\beta_{7 u v}{ }^{*} \ln \left(1+\right.$ intens_exp $\left.{ }_{u v i}\right)+\varepsilon_{u v i t}$

Ecuación para la variable número de empleados:

$$
\begin{aligned}
& \ln \left(1+\text { trabele }_{u v i t}\right)=\alpha+\alpha_{u}+\alpha_{u v}+\alpha_{u v i}+\beta_{1 u v} * t+\beta_{2 u v} * D_{i}+ \\
& \beta_{3 u v} * D_{i} * t+\beta_{4 u v} * \ln \left(1+e d_{a d}\right)+\beta_{5 u v} * \ln \left(1+\exp _{u v t} t o t_{u v i t}\right)+ \\
& \beta_{6 u v} * \ln \left(1+\text { productiv } v_{u v i t}\right)+\beta_{7 u v} * \ln \left(1+\text { intens_exp } \operatorname{expit}_{u v}\right)
\end{aligned}
$$


Ecuación para la variable productividad:

$\ln \left(1+\right.$ productiv $\left._{u v i t}\right)=\alpha+\alpha_{u}+\alpha_{u v}+\alpha_{u v i}+\beta_{1 u v} * t+\beta_{2 u v} * D_{i}+$ $\beta_{3 u v} * D_{i} * t+\beta_{4 u v} * \ln \left(1+e d a d_{u v i t}\right)+\beta_{5 u v} * \ln \left(1+\exp _{-} t o t_{u v i t}\right)+$ $\beta_{6 u v} * \ln \left(1+c n_{u v i t}\right)+\beta_{7 u v} * \ln \left(1+\right.$ trabele $\left._{u v i t}\right)+\varepsilon_{u v i t}$

En todas las especificaciones figuran las siguientes variables:

$t$ : esta variable representa el tiempo. Como nos interesa la variación entre 2014 y 2018, hemos contemplado solo el año inicial y el año final, sin tener en cuenta los años intermedios. Por lo tanto, $t=0$ para el año 2014 y $t=1$ para el año 2018. Su coeficiente $\beta_{1 u v}$ implica la diferencia atribuible al paso del tiempo para todas las empresas, independientemente del resto de factores, incluido haber recibido apoyo o no. En las tablas que recogen los resultados esta variable ha sido llamada «tiempo».

$D$ : esta variable representa haber recibido apoyo de ICEX en algún año del periodo 2014 , 2018. $D=0$ para las empresas que no han recibido apoyo en el periodo y $D=1$ para las empresas que sí lo han recibido. Su coeficiente $\beta_{2 u v}$ implica la diferencia atribuible a las empresas que han recibido apoyo, independientemente del resto de factores, incluido el momento del tiempo, por lo que indica una diferencia entre ambas poblaciones, lo que puede asimilarse a un sesgo de selección.

$D * t$ : esta variable representa haber recibido el apoyo de ICEX y encontrarse después del apoyo del mismo, es decir, $D * t=0$ solo cuando se cumple a la vez que $D=1$ y que $t=1$, y vale 0 en todos los demás casos. Su coeficiente $\beta_{3 u v}$ implica la diferencia atribuible al apoyo de ICEX, independientemente del resto de factores, incluido el posible sesgo de selección de las empresas que han recibido el apoyo y el paso del tiempo. En las tablas que recogen los resultados, esta variable ha sido llamada «apoyo».
Los subíndices indican de qué dependen las mediciones y los coeficientes. En particular:

- El subíndice $u$ indica el clúster de pertenencia de la empresa. Puede tener el valor de «todas» cuando se ha considerado toda la población sin distinción.

- El subíndice $v$ indica si la empresa ha recibido apoyo antes de 2014 o no. Puede tener el valor de «todas» cuando se ha considerado toda la población sin distinción.

- El subíndice $i$ indica cada empresa en particular.

- El subíndice $t$ indica el momento del tiempo.

Este conjunto de subíndices nos permite estimar los parámetros de forma independiente para el conjunto de empresas, para cada clúster por separado y para aquellas empresas que son veteranas y para las que no lo son. Esta es la razón por la que los parámetros se ven afectados por los subíndices $u$ y $v$.

En todas las especificaciones se ha evitado incluir como regresores aquellas variables que se pueden calcular a partir de otros regresores. Por ejemplo, si en la especificación figura el número de trabajadores y la productividad, hemos eliminado la cifra de negocios, pues hay una relación clara entre las tres variables.

Las Tablas 3 a 6 recogen los resultados significativos de estas estimaciones, tanto para todas las empresas como por tipo de empresa de forma individual, para las variables explicativas más relevantes, que incluyen el tiempo, el apoyo de ICEX, la exportación de bienes y la productividad.

Estos resultados muestran el incremento porcentual entre 2014 y 2018 en cada variable de interés atribuible al apoyo de ICEX, y vemos que los aumentos son diferentes para cada tipo de empresa, e incluso hay diferencias en función $\triangleright$ 
TABLA 3

ANÁLISIS DE CONTRIBUCIÓN POR TIPO DE EMPRESA PARA EL AUMENTO DE EXPORTACIÓN DE BIENES

\begin{tabular}{|c|c|c|c|c|c|c|c|c|c|}
\hline & \multicolumn{3}{|c|}{ Todas } & \multicolumn{3}{|c|}{ Veteranas } & \multicolumn{3}{|c|}{ No veteranas } \\
\hline & Tiempo & Apoyo & Product. & Tiempo & Apoyo & Product. & Tiempo & Apoyo & Product. \\
\hline Todas las empresas & $-0,07$ & 0,12 & 0,16 & $-0,06$ & $-0,16$ & 0,14 & $-0,07$ & 0,39 & 0,15 \\
\hline $\begin{array}{l}\text { Microempresas productivas de más } \\
\text { de } 10 \text { años de vida y con tímida } \\
\text { presencia en el exterior }\end{array}$ & $-0,047$ & 0,22 & 0,11 & $-0,04$ & & 0,11 & $-0,04$ & 0,39 & 0,11 \\
\hline $\begin{array}{l}\text { Microempresas poco productivas de } \\
\text { menos de } 10 \text { años de vida y tímida } \\
\text { presencia en el exterior }\end{array}$ & & & 0,03 & & & 0,03 & & & 0,04 \\
\hline $\begin{array}{l}\text { Empresas medianas y grandes de } \\
\text { más de } 15 \text { años de vida muy } \\
\text { productivas y volcadas en el exterior }\end{array}$ & $-0,15$ & & 0,39 & $-0,14$ & $-0,15$ & 0,36 & $-0,15$ & 0,25 & 0,36 \\
\hline $\begin{array}{l}\text { Microempresas de reciente creación } \\
\text { muy productivas y volcadas en el } \\
\text { exterior }\end{array}$ & $-0,09$ & 1,06 & 0,14 & $-0,08$ & & 0,13 & $-0,09$ & 1,17 & 0,14 \\
\hline $\begin{array}{l}\text { Microempresas de más de } 10 \text { años de } \\
\text { vida muy productivas y muy volcadas } \\
\text { en el exterior }\end{array}$ & $-0,08$ & & 0,25 & $-0,07$ & $-0,57$ & 0,24 & $-0,08$ & & 0,25 \\
\hline $\begin{array}{l}\text { Pequeñas empresas de más de } 12 \\
\text { años de vida volcadas en el exterior }\end{array}$ & $-0,047$ & & & $-0,04$ & & & $-0,05$ & & \\
\hline $\begin{array}{l}\text { Microempresas de menos de } 10 \text { años } \\
\text { de vida con baja productividad y } \\
\text { tímida presencia en el exterior }\end{array}$ & $-0,047$ & 0,87 & 0,06 & $-0,04$ & 0,83 & 0,05 & 0,04 & 0,89 & 0,05 \\
\hline \multicolumn{10}{|c|}{$\begin{array}{l}\text { Variable dependiente: exportación de bienes (en logaritmo). Técnica: diferencias en diferencias, regresión por datos de panel. Solo se muestran aquellos } \\
\text { resultados significativos. Los valores implican incrementos porcentuales de la exportación de bienes entre } 2014 \text { y } 2018 \text {. }\end{array}$} \\
\hline
\end{tabular}

TABLA 4

ANÁLISIS DE CONTRIBUCIÓN POR TIPO DE EMPRESA PARA EL AUMENTO DE LA CIFRA DE NEGOCIOS

\begin{tabular}{|c|c|c|c|c|c|c|c|c|c|}
\hline & \multicolumn{3}{|c|}{ Todas } & \multicolumn{3}{|c|}{ Veteranas } & \multicolumn{3}{|c|}{ No veteranas } \\
\hline & Tiempo & Apoyo & Product. & Tiempo & Apoyo & Product. & Tiempo & Apoyo & Product. \\
\hline Todas las empresas & $-0,004$ & 0,09 & 0,82 & & 0,04 & 0,82 & 0,00 & 0,13 & 0,81 \\
\hline $\begin{array}{l}\text { Microempresas productivas de más } \\
\text { de } 10 \text { años de vida y con tímida } \\
\text { presencia en el exterior }\end{array}$ & & 0,10 & 0,77 & & & 0,78 & & 0,14 & 0,77 \\
\hline $\begin{array}{l}\text { Microempresas poco productivas de } \\
\text { menos de } 10 \text { años de vida y tímida } \\
\text { presencia en el exterior }\end{array}$ & & & 0,99 & & & 0,99 & & & 0,99 \\
\hline $\begin{array}{l}\text { Empresas medianas y grandes de } \\
\text { más de } 15 \text { años de vida muy } \\
\text { productivas y volcadas en el exterior }\end{array}$ & & 0,07 & 0,84 & & 0,06 & 0,84 & & 0,10 & 0,84 \\
\hline $\begin{array}{l}\text { Microempresas de reciente creación } \\
\text { muy productivas y volcadas en el } \\
\text { exterior }\end{array}$ & & 0,32 & 0,80 & & & 0,80 & & 0,32 & 0,80 \\
\hline $\begin{array}{l}\text { Microempresas de más de } 10 \text { años de } \\
\text { vida muy productivas y muy volcadas } \\
\text { en el exterior }\end{array}$ & 0,12 & 0,18 & 0,81 & 0,12 & 0,12 & 0,81 & 0,12 & 0,22 & 0,81 \\
\hline $\begin{array}{l}\text { Pequeñas empresas de más de } 12 \\
\text { años de vida volcadas en el exterior }\end{array}$ & 7,98 & 5,31 & & 7,97 & 5,76 & & 7,98 & 4,89 & \\
\hline $\begin{array}{l}\text { Microempresas de menos de } 10 \text { años } \\
\text { de vida con baja productividad y } \\
\text { tímida presencia en el exterior }\end{array}$ & $-0,13$ & 0,13 & 0,92 & $-0,12$ & & 0,92 & $-0,13$ & 0,14 & 0,92 \\
\hline \multicolumn{10}{|c|}{$\begin{array}{l}\text { Variable dependiente: cifra de negocios (en logaritmo). Técnica: diferencias en diferencias, regresión por datos de panel. Solo se muestran aquellos } \\
\text { resultados significativos. Los valores implican incrementos porcentuales de la cifra de negocios entre } 2014 \text { y } 2018 . \\
\text { Veteranas: empresas que han tenido apoyo de ICEX antes de } 2014 \text {. } \\
\text { No veteranas: empresas que no han tenido apoyo de ICEX antes de } 2014 .\end{array}$} \\
\hline Fuente: CEEPE y elaboración propia. & & & & & & & & & \\
\hline
\end{tabular}




\section{EL IMPACTO A CORTO Y MEDIO PLAZO DEL APOYO DE ICEX}

TABLA 5

ANÁLISIS DE CONTRIBUCIÓN POR TIPO DE EMPRESA PARA EL AUMENTO DEL NÚMERO DE EMPLEADOS

\begin{tabular}{|c|c|c|c|c|c|c|c|c|c|}
\hline & \multicolumn{3}{|c|}{ Todas } & \multicolumn{3}{|c|}{ Veteranas } & \multicolumn{3}{|c|}{ No veteranas } \\
\hline & Tiempo & Apoyo & Product. & Tiempo & Apoyo & Product. & Tiempo & Apoyo & Product. \\
\hline Todas las empresas & 0,004 & 0,09 & $-0,13$ & 0,005 & 0,05 & $-0,13$ & 0,004 & 0,13 & $-0,13$ \\
\hline $\begin{array}{l}\text { Microempresas productivas de más } \\
\text { de } 10 \text { años de vida y con tímida } \\
\text { presencia en el exterior }\end{array}$ & 0,00 & 0,09 & $-0,16$ & 0,00 & 0,04 & $-0,16$ & 0,00 & 0,12 & $-0,16$ \\
\hline $\begin{array}{l}\text { Microempresas poco productivas de } \\
\text { menos de } 10 \text { años de vida y tímida } \\
\text { presencia en el exterior }\end{array}$ & & & $-0,02$ & & & $-0,02$ & & & $-0,02$ \\
\hline $\begin{array}{l}\text { Empresas medianas y grandes de } \\
\text { más de } 15 \text { años de vida muy } \\
\text { productivas y volcadas en el exterior }\end{array}$ & & 0,07 & $-0,15$ & & 0,05 & $-0,15$ & & 0,09 & $-0,15$ \\
\hline $\begin{array}{l}\text { Microempresas de reciente creación } \\
\text { muy productivas y volcadas en el } \\
\text { exterior }\end{array}$ & & 0,27 & $-0,14$ & & & $-0,14$ & & 0,27 & $-0,14$ \\
\hline $\begin{array}{l}\text { Microempresas de más de } 10 \text { años de } \\
\text { vida muy productivas y muy volcadas } \\
\text { en el exterior }\end{array}$ & 0,08 & 0,17 & $-0,14$ & 0,08 & 0,13 & $-0,14$ & 0,08 & 0,21 & $-0,14$ \\
\hline $\begin{array}{l}\text { Pequeñas empresas de más de } 12 \\
\text { años de vida volcadas en el exterior }\end{array}$ & $-0,11$ & 0,24 & & $-0,11$ & & & $-0,11$ & 0,35 & \\
\hline $\begin{array}{l}\text { Microempresas de menos de } 10 \text { años } \\
\text { de vida con baja productividad y } \\
\text { tímida presencia en el exterior }\end{array}$ & $-0,08$ & 0,11 & $-0,06$ & $-0,08$ & & $-0,06$ & $-0,08$ & 0,12 & $-0,06$ \\
\hline \multicolumn{10}{|c|}{$\begin{array}{l}\text { Variable dependiente: número de empleados (en logaritmo). Técnica: diferencias en diferencias, regresión por datos de panel. Solo se muestran aquellos } \\
\text { resultados significativos. Los valores implican incrementos porcentuales del número de empleados entre } 2014 \text { y } 2018 . \\
\text { Veteranas: empresas que han tenido apoyo de ICEX antes de } 2014 \text {. } \\
\text { No veteranas: empresas que no han tenido apoyo de ICEX antes de } 2014 \text {. }\end{array}$} \\
\hline \multicolumn{10}{|l|}{ Fuente: CEEPE y elaboración propia. } \\
\hline
\end{tabular}

TABLA 6

ANÁLISIS DE CONTRIBUCIÓN POR TIPO DE EMPRESA PARA EL AUMENTO DE LA PRODUCTIVIDAD

\begin{tabular}{|c|c|c|c|c|c|c|c|c|c|c|c|c|}
\hline & \multicolumn{4}{|c|}{ Todas } & \multicolumn{4}{|c|}{ Veteranas } & \multicolumn{4}{|c|}{ No veteranas } \\
\hline & Tiempo & Apoyo & $\begin{array}{c}\text { Cifra } \\
\text { negocios }\end{array}$ & Export & Tiempo & Apoyo & $\begin{array}{c}\text { Cifra_ } \\
\text { negocios }\end{array}$ & Export & Tiempo & Apoyo & $\begin{array}{c}\text { Cifra } \\
\text { negocios }\end{array}$ & Export \\
\hline Todas las empresas & 0,009 & 0,02 & 1,00 & 0,00 & 0,009 & 0,02 & 1,00 & 0,00 & 0,009 & 0,027 & 1,00 & 0,00 \\
\hline $\begin{array}{l}\text { Microempresas productivas de más } \\
\text { de } 10 \text { años de vida y con tímida } \\
\text { presencia en el exterior }\end{array}$ & 0,005 & 0,02 & 1,00 & 0,00 & 0,005 & 0,02 & 1,00 & 0,00 & 0,005 & 0,03 & 1,00 & 0,00 \\
\hline $\begin{array}{l}\text { Microempresas poco productivas } \\
\text { de menos de } 10 \text { años de vida y } \\
\text { tímida presencia en el exterior }\end{array}$ & 0,08 & & 0,98 & & 0,08 & & 0,98 & & 0,08 & & 0,98 & \\
\hline $\begin{array}{l}\text { Empresas medianas y grandes de } \\
\text { más de } 15 \text { años de vida muy } \\
\text { productivas y volcadas en el } \\
\text { exterior }\end{array}$ & 0,01 & & 0,99 & & 0,01 & & 0,99 & & 0,11 & 0,003 & 0,98 & \\
\hline $\begin{array}{l}\text { Microempresas de reciente } \\
\text { creación muy productivas y } \\
\text { volcadas en el exterior }\end{array}$ & $-0,02$ & 0,04 & 1,00 & 0,001 & $-0,02$ & & 1,00 & 0,00 & $-0,02$ & 0,04 & 1,00 & 0,001 \\
\hline $\begin{array}{l}\text { Microempresas de más de } 10 \text { años } \\
\text { de vida muy productivas y muy } \\
\text { volcadas en el exterior }\end{array}$ & $-0,01$ & 0,06 & 1,00 & 0,00 & $-0,01$ & 0,05 & 0,99 & & $-0,01$ & 0,06 & 0,99 & 0,00 \\
\hline $\begin{array}{l}\text { Pequeñas empresas de más de } 12 \\
\text { años de vida volcadas en el exterior }\end{array}$ & 6,94 & 3,74 & & 0,08 & 6,94 & 4,24 & & 0,08 & 6,94 & 3,25 & & 0,09 \\
\hline $\begin{array}{l}\text { Microempresas de menos de } 10 \\
\text { años de vida con baja productividad } \\
\text { y tímida presencia en el exterior }\end{array}$ & 0,24 & 0,01 & 1,00 & & 0,02 & & 0,99 & & 0,02 & 0,014 & 0,99 & \\
\hline \multicolumn{13}{|c|}{$\begin{array}{l}\text { Variable dependiente: productividad (en logaritmo). Técnica: diferencias en diferencias, regresión por datos de panel. Solo se muestran aquellos resulta- } \\
\text { dos significativos. Los valores implican incrementos porcentuales de la productividad entre } 2014 \text { y } 2018 \text {. }\end{array}$} \\
\hline \multicolumn{13}{|l|}{ Fuente: CEEPE y elaboración propia. } \\
\hline
\end{tabular}


de si nos utilizaba por primera vez en el periodo o nos había utilizado antes. Dejamos la interpretación de estos resultados para el apartado de conclusiones y recomendaciones, pues para interpretarlos mejor tenemos que conocer cómo evolucionan estos efectos a partir del apoyo.

\section{Estimación del retorno de inversión del apoyo de ICEX}

A partir de estos resultados, hemos realizado un primer cálculo de forma aproximativa del retorno del presupuesto público gestionado por ICEX y dedicado al apoyo a la internacionalización de la empresa contemplando los tipos impositivos medios del impuesto de sociedades y del IRPF. En este cálculo contemplamos el presupuesto en su conjunto sin descontar ninguna partida específica, si bien no incluye, por razones evidentes, el que no gestiona la institución directamente, como una parte importante del funcionamiento de las Oficinas Económicas y Comerciales de España en el exterior y de la red territorial.

En concreto, para el incremento en las cifras de exportación de bienes, cifra de negocios o número de empleados, se ha calculado el valor que se obtendría para la cifra correspondiente y conocida en 2014 de las empresas que han recibido apoyo durante el periodo en las ecuaciones anteriores sustituyendo el valor del coeficiente estimado y suponiendo todos los demás factores constantes. Por ejemplo, para la exportación de bienes, las expresiones para el incremento esperado debido al apoyo de ICEX y debido a un incremento de la productividad, ignorando todos los demás efectos, serían:

Estimación de la exportación de bienes debida exclusivamente al apoyo para 2018:

$$
\operatorname{exp\_ tot_{2018}}=\left(1+\operatorname{exp\_ tot}{ }_{2014}\right) * \mathrm{e}^{\beta_{3}}-1
$$

Estimación de la exportación de bienes debida exclusivamente a un incremento $p$ en la productividad para 2018:

$$
\text { exp_tot }{ }_{2018}=\left(1+e x p \_t o t_{2014}\right) * \mathrm{e}^{(1+p) * \beta_{6}}-1
$$

Para el cálculo del retorno de la inversión mediante incremento en la recaudación por las cifras de exportación de bienes y de negocio se ha tenido en cuenta el tipo de gravamen general del impuesto de sociedades para periodos impositivos iniciados a partir del 1 de enero de 2016 , que es del $25 \%$. Para una mejor aproximación se han aplicado coeficientes correctores a las cifras de exportaciones y de negocios para descontar el valor añadido importado ${ }^{2}$ que se incorpora a las exportaciones o a la cifra de negocios. El supuesto implícito es que el valor añadido de origen nacional adquirido por las empresas que reciben apoyo de ICEX para exportar o incrementar su cifra de negocios se ve sometido a un gravamen similar, bien a través del impuesto de sociedades aplicado a los proveedores, bien a través del IRPF (en el caso de proveedores autónomos o en el caso de los salarios).

Por su parte, para el cálculo del retorno de la inversión mediante incremento en la recaudación por el aumento del empleo se ha partido del salario medio bruto en España para el año 2018, que fue de 26.922,00 euros, sobre el que se ha aplicado una estimación del gravamen medio del IRPF para ese año. Dado que la distribución del gravamen está muy sesgada hacia los valores bajos de la escala, el $\triangleright$

2 En el caso de las exportaciones, se ha aplicado un coeficiente corrector de 0,77 para estimar el contenido de valor añadido de origen español. Para el cálculo de esta cifra se han utilizado datos de la base de datos Trade in Value Added (TiVA) de la Organización para la Cooperación y Desarrollo Económico (OCDE), correspondientes a la media de 2014 y 2015, años del periodo de análisis para los que se dispone de información. En el caso de la cifra de negocios se ha aplicado un coeficiente corrector de 0,69 , obtenido a partir de la Contabilidad Nacional Anual de España que publica el INE, con datos del periodo 2014 a 2018. 
TABLA 7

IMPACTO PRESUPUESTARIO DE LA ACTIVIDAD DE ICEX EN EXPORTACIÓN DE BIENES, CIFRA DE NEGOCIOSY RETRIBUCIÓN DEL EMPLEO

\begin{tabular}{|c|c|c|c|}
\hline \multirow[b]{2}{*}{ Variable económica } & \multicolumn{3}{|c|}{ Impacto presupuestario } \\
\hline & $\begin{array}{l}\text { Límite } \\
\text { inferior } \\
\text { (IC 95\%) }\end{array}$ & $\begin{array}{l}\text { Estimación } \\
\text { puntual }\end{array}$ & $\begin{array}{l}\text { Límite } \\
\text { superior } \\
\text { (IC 95\%) }\end{array}$ \\
\hline $\begin{array}{l}\text { INCREMENTO DE EXPORTACIÓN / PRESUPUESTO PARA TODO EL PERIODO } \\
\text { (euros de exportación adicional por euro de presupuesto ICEX) }\end{array}$ & $12,72 €$ & $26,22 €$ & $43,04 €$ \\
\hline INCREMENTO DE RECAUDACIÓN POR TRIBUTACIÓN (1) & $2,45 €$ & $5,05 €$ & $8,28 €$ \\
\hline $\begin{array}{l}\text { INCREMENTO DE EXPORTACIÓN / PRESUPUESTO POR AÑO } \\
\text { (euros de exportación adicional anual por euro de presupuesto ICEX) }\end{array}$ & $2,54 €$ & $5,24 €$ & $8,61 €$ \\
\hline INCREMENTO DE RECAUDACIÓN POR TRIBUTACIÓN (1) & $0,49 €$ & $1,01 €$ & $1,66 €$ \\
\hline $\begin{array}{l}\text { INCREMENTO DE LA CIFRA DE NEGOCIOS / PRESUPUESTO PARA TODO EL PERIODO } \\
\text { (euros de facturación adicional por euro de presupuesto ICEX) }\end{array}$ & $90,33 €$ & $102,13 €$ & $114,06 €$ \\
\hline INCREMENTO DE RECAUDACIÓN POR TRIBUTACIÓN (2) & $15,58 €$ & $17,62 €$ & $19,68 €$ \\
\hline $\begin{array}{l}\text { INCREMENTO DE LA CIFRA DE NEGOCIOS / PRESUPUESTO POR AÑO } \\
\text { (euros de facturación adicional anual por euro de presupuesto ICEX) }\end{array}$ & $18,07 €$ & $20,43 €$ & $22,81 €$ \\
\hline INCREMENTO DE RECAUDACIÓN POR TRIBUTACIÓN (2) & $3,12 €$ & $3,52 €$ & $3,94 €$ \\
\hline $\begin{array}{l}\text { INCREMENTO DE RETRIBUCIÓN / PRESUPUESTO PARA TODO EL PERIODO } \\
\text { (euros de salario bruto adicional por euro de presupuesto ICEX) }\end{array}$ & $7,48 €$ & $8,45 €$ & $9,44 €$ \\
\hline INCREMENTO DE RECAUDACIÓN POR TRIBUTACIÓN & $1,88 €$ & $2,13 €$ & $2,38 €$ \\
\hline $\begin{array}{l}\text { INCREMENTO DE RETRIBUCIÓN / PRESUPUESTO POR AÑO } \\
\text { (euros de salario bruto adicional anual por euro de presupuesto ICEX) }\end{array}$ & $1,50 €$ & $1,69 €$ & $1,89 €$ \\
\hline INCREMENTO DE RECAUDACIÓN POR TRIBUTACIÓN & $0,38 €$ & $0,43 €$ & $0,48 €$ \\
\hline \multicolumn{4}{|c|}{$\begin{array}{l}\text { Los valores en negro representan incrementos anualizados en euros en la variable considerada atribuible a cada euro del presupuesto de ICEX. } \\
\text { Los valores en rojo representan el retorno de inversión anualizado en euros atribuible a cada euro de presupuesto de ICEX. } \\
\text { La columna central muestra la estimación puntual de los valores, mientras que las otras dos columnas representan el límite inferior y el límite superior } \\
\text { para esos valores, con una confianza del } 95 \% \text {. } \\
\text { (1) Para el retorno de inversión por el incremento de las exportaciones se ha aplicado un coeficiente corrector de } 0,77 \text {, para estimar el contenido de } \\
\text { valor añadido de origen español. Para el cálculo de esta cifra se han utilizado datos de la base de datos Trade in Value Added (TiVA) de la OCDE, } \\
\text { correspondientes a la media de } 2014 \text { y } 2015 \text {, años del periodo de análisis para los que se dispone de información. } \\
\text { (2) Para el retorno de inversión por el incremento de la cifra de negocios se ha aplicado un coeficiente corrector de } 0,69 \text {, obtenido a partir de la } \\
\text { Contabilidad Nacional Anual de España que publica el INE, con datos del periodo } 2014 \text { a } 2018 \text {. }\end{array}$} \\
\hline
\end{tabular}

gravamen medio se ha estimado como el valor que excede del tipo mínimo en un $20 \%$ del rango entre el tipo mínimo y el máximo, es decir, el $25,20 \%$.

La Tabla 7 recoge los cálculos estimados del incremento global que el presupuesto de ICEX ha tenido en la exportación de bienes, la cifra de negocios y la retribución de las empresas que han recibido apoyos de ICEX durante el periodo 2014-2018, teniendo en cuenta que todo lo demás no cambia y sin tener en cuenta las caídas producidas por otros factores. Nuestro interés radica más en la comparación del efecto debido a ICEX en relación con el efecto debido a la productividad que en las cifras absolutas calculadas, que ni pueden ni deben suplir un ejercicio más robusto realizado con los microdatos fiscales de empresas y empleados.

El apoyo agregado de ICEX tiene un efecto notable en el incremento de la exportación de bienes de la empresa receptora del apoyo. En el peor de los casos, se estima para cada euro del presupuesto global ejecutado de ICEX entre 2014 y 2018 un retorno vía impuestos por el incremento de la exportación de bienes atribuible a ICEX en las empresas receptoras de los apoyos de 2,45 euros a lo largo del periodo. En términos de cifra de negocio, que incluye por definición la exportación de bienes, este $\square$ 


\section{EL IMPACTO A CORTO Y MEDIO PLAZO DEL APOYO DE ICEX}

TABLA 9

COMPARATIVA ENTRE EFECTO ICEXY EFECTO PRODUCTIVIDAD EN EL INCREMENTO DE LA CIFRA DE NEGOCIOS

\begin{tabular}{|c|c|c|c|}
\hline Incremento entre 2014 y 2018 & $\begin{array}{l}\text { Límite inferior IC } \\
\quad 95 \%\end{array}$ & $\begin{array}{l}\text { Estimación } \\
\text { puntual }\end{array}$ & $\begin{array}{l}\text { Límite superior } \\
\text { IC } 95 \%\end{array}$ \\
\hline \multicolumn{4}{|l|}{ Factor apoyo de ICEX } \\
\hline Coeficiente en ecuación logarítmica (técnica Dif-in-Dif) & 0,08 & 0,09 & 0,10 \\
\hline $\begin{array}{l}\text { Incremento total para empresas que han recibido apoyo de ICEX } \\
\text { durante el periodo }\end{array}$ & $49.492 .674 .696,57$ & $55.962 .315 .859,10$ & $62.496 .977 .996,29$ \\
\hline \multicolumn{4}{|l|}{ Factor incremento en productividad } \\
\hline $\begin{array}{l}\text { Coeficiente en ecuación logarítmica (técnica Dif-in-Dif) } \\
\text { IC } 95 \% \text { para la estimación: }[0.153,0.169]\end{array}$ & \multicolumn{3}{|c|}{0,82} \\
\hline $\begin{array}{l}\text { Incremento total para empresas por su aumento en productividad } \\
\text { durante el periodo }\end{array}$ & \multicolumn{3}{|c|}{$957.521 .505 .638,74$} \\
\hline \multicolumn{4}{|l|}{ Comparativa entre efecto ICEX y efecto productividad } \\
\hline Comparación incremento apoyo ICEX / incremento productividad & $5,17 \%$ & $5,84 \%$ & $6,53 \%$ \\
\hline $\begin{array}{l}\text { Incremento debido al apoyo de ICEX en relación con el incremento } \\
\text { debido a la combinación de ambos factores }\end{array}$ & $4,91 \%$ & $5,52 \%$ & $6,13 \%$ \\
\hline \multicolumn{4}{|c|}{$\begin{array}{l}\text { La columna central muestra la estimación puntual del incremento en la cifra de negocios, mientras que las otras dos columnas muestran el límite inferior } \\
\text { y el límite superior para ese valor, con una confianza del } 95 \% \text {. } \\
\text { Se ha calculado el incremento asociado en exclusiva a una mejora de la productividad del } 10 \% \text {, que es } 10 \text { veces mayor que el debido exclusivamente } \\
\text { al paso del tiempo en el periodo. }\end{array}$} \\
\hline \multicolumn{4}{|l|}{ Fuente: ICEX, CEEPE y elaboración propia. } \\
\hline
\end{tabular}

\section{Efecto del apoyo de ICEX sobre el empleo}

Los resultados muestran igualmente que el apoyo de ICEX se traduce en un incremento en el número de empleados que, aun así, no compensa la disminución de empleados asociada al incremento de la productividad, lo que es un reflejo del hecho contrastado en la literatura de que en España los incrementos de productividad están estadísticamente asociados a disminuciones del empleo ${ }^{3}$. La Tabla 10 muestra el efecto paliativo del apoyo de ICEX y el efecto neto del efecto combinado entre el apoyo de ICEX y el aumento de la productividad.

3 Ver, por ejemplo, Prados (2017) o Ariño (2017).

TABLA 10

INCREMENTO EN EL NÚMERO DE EMPLEADOS ENTRE 2014 Y 2018 ATRIBUIBLES AL APOYO DE ICEXY SU COMPARACIÓN CON LA DESTRUCCIÓN DE EMPLEO ASOCIADA AL AUMENTO DE PRODUCTIVIDAD

\begin{tabular}{|c|c|c|c|}
\hline Incremento entre 2014 y 2018 & $\begin{array}{l}\text { Límite inferior } \\
\text { IC } 95 \%\end{array}$ & $\begin{array}{l}\text { Estimación } \\
\text { puntual }\end{array}$ & $\begin{array}{l}\text { Límite superior } \\
\text { IC } 95 \%\end{array}$ \\
\hline $\begin{array}{l}\text { Incremento total para empresas que han recibido apoyo de ICEX durante } \\
\text { el periodo }\end{array}$ & $152.144,82$ & $172.033,06$ & $192.121,18$ \\
\hline $\begin{array}{l}\text { Incremento total para empresas por su aumento en productividad } \\
\text { durante el periodo }\end{array}$ & \multicolumn{3}{|c|}{$-272.503,65$} \\
\hline $\begin{array}{l}\text { Compensación incremento apoyo ICEX / destrucción por incremento de } \\
\text { productividad }\end{array}$ & $55,83 \%$ & $63,13 \%$ & $70,50 \%$ \\
\hline Creación de empleo neta & $-120.358,83$ & $-100.470,59$ & $-80.382,47$ \\
\hline \multicolumn{4}{|c|}{$\begin{array}{l}\text { La columna central muestra la estimación puntual del incremento en el número de empleados, mientras que las otras dos columnas muestran el límite } \\
\text { inferior y el límite superior para ese valor, con una confianza del } 95 \% \text {. } \\
\text { Se ha calculado el incremento asociado en exclusiva a una mejora de la productividad del } 10 \% \text {, que es } 10 \text { veces mayor que el debido exclusivamente } \\
\text { al paso del tiempo en el periodo. }\end{array}$} \\
\hline \multicolumn{4}{|c|}{ Fuente: ICEX, CEEPE y elaboración propia. } \\
\hline
\end{tabular}


Podemos afirmar que, en el peor escenario, el apoyo del ICEX hace que se destruya solo la mitad del empleo que cabría esperar de pérdida para una empresa que mantenga una moderada mejora de su productividad. En el escenario más favorable, el apoyo de ICEX permite que solo se pierda un $30 \%$ de empleo.

\section{Evolución de las variables de interés a partir del apoyo de ICEX}

En este apartado, tenemos en cuenta la variable llamada «desfase», que mide el tiempo en años transcurrido desde el inicio del apoyo de ICEX en el periodo 2014-2018 hasta el último año del periodo, que es 2018. Esto nos permite ver el efecto en el tiempo que se produce en la empresa tras el apoyo de ICEX, si bien este efecto puede no tener como causa el propio apoyo de ICEX, ya que puede deberse a algún sesgo de selección de las empresas que recurren a la institución. Combinando estos resultados con el efecto atribuible al apoyo, que hemos mostrado en el apartado anterior, podemos hacernos una imagen más clara de cómo contribuye el apoyo de ICEX a la marcha de estas empresas. Asimismo, hay que tener en cuenta que, de acuerdo con todas las evaluaciones que hemos realizado internamente mediante la técnica del falso contrafactual, cabe esperar un efecto entre uno y dos años posterior al apoyo.

En primer lugar, el Gráfico 6 muestra el volumen de exportación de bienes en 2018 de las empresas apoyadas en función de su tipo de empresa, del tiempo transcurrido desde el inicio del apoyo y de si habían recibido o no apoyo previamente. Al no haber diferencia entre los tipos de empresa que entran año a año, cada valor de desfase puede considerarse como identificador de una cohorte del tipo de empresa en cuestión, y por lo tanto la lectura de este gráfico muestra cómo $\triangleright$

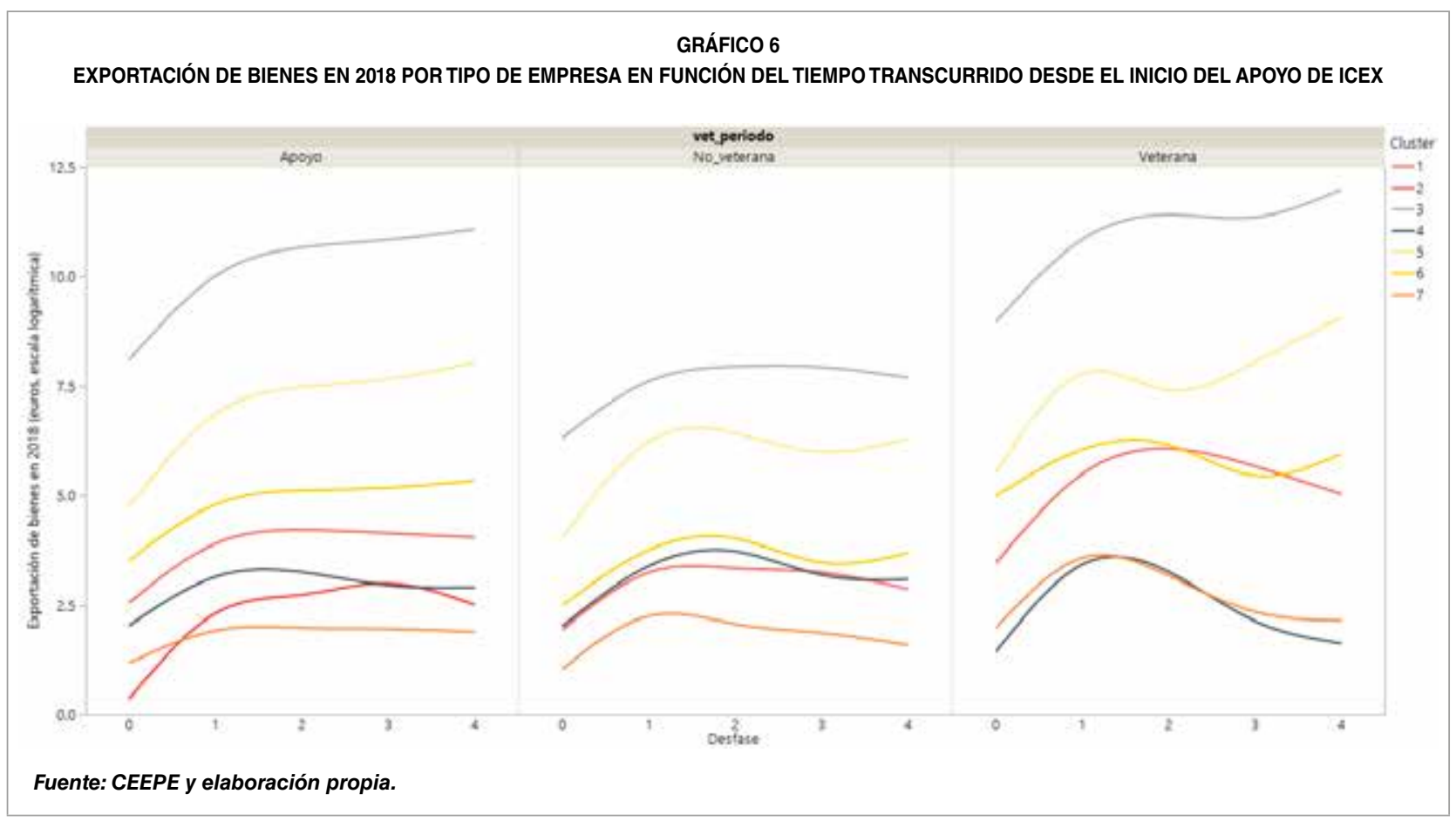


evoluciona la exportación de estas empresas en función del tiempo transcurrido desde el apoyo.

Hemos sometido estos datos de evolución por tipo de empresa y estatus de veteranía a un análisis de datos funcionales, cuyos detalles pueden verse en el informe completo. Este análisis muestra dos grupos claros. Un primer grupo se asocia a empresas con una cierta experiencia exterior y una alta productividad que experimentan un considerable incremento el primer año tras el apoyo, si bien tras el tercer año este incremento es ligeramente menor del esperable. El segundo grupo se asocia a empresas con poca experiencia internacional que solicitan el apoyo en pleno crecimiento en sus cifras de exportación de bienes, que aumentan igualmente de forma considerable el primer año tras el apoyo, no obstante, tras el tercer año este incremento es ligeramente menor del esperable.

\section{Análisis de varianza del incremento en los valores de las variables de interés según el tiempo transcurrido desde el inicio del apoyo}

Tras el análisis del punto anterior, descriptivo y exploratorio, queremos saber si los diferentes valores observados a lo largo del tiempo son significativamente distintos entre sí. Para ello, hemos llevado a cabo un análisis de varianza de las variables de interés por el tiempo transcurrido desde el inicio del apoyo. En particular, se ha utilizado el test del rango múltiple de Duncan sobre la diferencia del valor de la variable entre 2014 y 2018 tomada en logaritmo. Las Tablas 11 a 15 muestran los resultados de este análisis. En todas ellas, colores distintos muestran valores que son significativamente distintos entre sí. Los tonos verdes indican valores significativamente mayores que 0 y los tonos rojos, valores significativamente menores que $0 . \quad \square$

TABLA 11

EFECTO OBSERVADO EN EXPORTACIÓN DE BIENES POR TIPO DE EMPRESA SEGÚN EL TIEMPO TRANSCURRIDO DESDE EL INICIO DEL APOYO DE ICEX

\begin{tabular}{|c|c|c|c|c|c|}
\hline \multirow{2}{*}{ Tipo de empresa } & \multicolumn{5}{|c|}{ Desfase desde inicio del apoyo (años) } \\
\hline & 0 & 1 & 2 & 3 & 4 \\
\hline Todas & 0,04 & 1,46 & 0,85 & 0,11 & $-0,40$ \\
\hline $\begin{array}{l}\text { Microempresas productivas de más de } 10 \text { años de vida y con tímida presencia en el } \\
\text { exterior }\end{array}$ & $-0,04$ & 0,63 & 0,40 & $-0,04$ & $-0,32$ \\
\hline $\begin{array}{l}\text { Microempresas poco productivas de menos de } 10 \text { años de vida y tímida presencia en el } \\
\text { exterior }\end{array}$ & 0,01 & 1,55 & 0,33 & 0,72 & $-0,47$ \\
\hline $\begin{array}{l}\text { Empresas medianas y grandes de más de } 15 \text { años de vida muy productivas y volcadas } \\
\text { en el exterior }\end{array}$ & $-0,13$ & 0,39 & 0,22 & $-0,19$ & $-0,46$ \\
\hline Microempresas de reciente creación muy productivas y volcadas en el exterior & 0,05 & 1,82 & 1,86 & 0,87 & 0,30 \\
\hline $\begin{array}{l}\text { Microempresas de más de } 10 \text { años de vida muy productivas y muy volcadas en el } \\
\text { exterior }\end{array}$ & $-0,04$ & 0,96 & 0,29 & $-0,21$ & $-0,81$ \\
\hline Pequeñas empresas de más de 12 años de vida volcadas en el exterior & $-0,01$ & $-0,07$ & 0,74 & $-0,90$ & 0,02 \\
\hline $\begin{array}{l}\text { Microempresas de menos de } 10 \text { años de vida con baja productividad y tímida presencia } \\
\text { en el exterior }\end{array}$ & 0,00 & 1,37 & 0,66 & 0,43 & 0,23 \\
\hline \multicolumn{6}{|c|}{$\begin{array}{l}\text { En desfase } 0 \text { se encuentran tanto las empresas que no han recibido apoyo de ICEX en el periodo como las que recibieron apoyo por primera vez en } 2018 \text {. } \\
\text { Colores distintos muestran valores que son significativamente distintos entre sí. Los tonos verdes indican valores significativamente mayores que } 0 \text { y los } \\
\text { tonos rojos, valores significativamente menores que } 0 \text {. } \\
\text { Los coeficientes implican incrementos porcentuales de la exportación de bienes entre } 2014 \text { y } 2018 \text {. }\end{array}$} \\
\hline \multicolumn{6}{|l|}{ Fuente: CEEPE y elaboración propia. } \\
\hline
\end{tabular}


TABLA 12

EFECTO OBSERVADO EN CIFRA DE NEGOCIOS POR TIPO DE EMPRESA SEGÚN EL TIEMPO TRANSCURRIDO DESDE EL INICIO DEL APOYO DE ICEX

\begin{tabular}{|c|c|c|c|c|c|}
\hline \multirow{2}{*}{ Tipo de empresa } & \multicolumn{5}{|c|}{ Desfase desde inicio del apoyo (años) } \\
\hline & 0 & 1 & 2 & 3 & 4 \\
\hline Todas & 3,00 & 3,56 & 1,72 & 0,93 & 0,40 \\
\hline $\begin{array}{l}\text { Microempresas productivas de más de } 10 \text { años de vida y con tímida presencia en el } \\
\text { exterior }\end{array}$ & $-0,28$ & 0,29 & 0,02 & $-0,15$ & $-0,24$ \\
\hline $\begin{array}{l}\text { Microempresas poco productivas de menos de } 10 \text { años de vida y tímida presencia en el } \\
\text { exterior }\end{array}$ & 9,75 & 10,62 & 10,39 & 11,98 & 10,73 \\
\hline $\begin{array}{l}\text { Empresas medianas y grandes de más de } 15 \text { años de vida muy productivas y volcadas } \\
\text { en el exterior }\end{array}$ & $-0,28$ & 0,21 & 0,04 & $-0,04$ & $-0,05$ \\
\hline Microempresas de reciente creación muy productivas y volcadas en el exterior & 0,83 & 2,70 & 1,83 & 1,21 & 0,37 \\
\hline $\begin{array}{l}\text { Microempresas de más de } 10 \text { años de vida muy productivas y muy volcadas en el } \\
\text { exterior }\end{array}$ & 0,16 & 0,53 & 0,02 & $-0,15$ & $-0,26$ \\
\hline Pequeñas empresas de más de 12 años de vida volcadas en el exterior & 11,33 & 13,02 & 13,66 & 13,49 & 13,51 \\
\hline $\begin{array}{l}\text { Microempresas de menos de } 10 \text { años de vida con baja productividad y tímida presencia } \\
\text { en el exterior }\end{array}$ & $-0,29$ & 1,52 & 1,01 & 0,60 & $-0,35$ \\
\hline \multicolumn{6}{|c|}{$\begin{array}{l}\text { En desfase } 0 \text { se encuentran tanto las empresas que no han recibido apoyo de ICEX en el periodo como las que recibieron apoyo por primera vez en } 2018 . \\
\text { Colores distintos muestran valores que son significativamente distintos entre sí. Los tonos verdes indican valores significativamente mayores que } 0 \text { y los } \\
\text { tonos rojos, valores significativamente menores que } 0 \text {. } \\
\text { Los coeficientes implican incrementos porcentuales de la cifra de negocios entre } 2014 \text { y } 2018 \text {. }\end{array}$} \\
\hline \multicolumn{6}{|l|}{ Fuente: CEEPE y elaboración propia. } \\
\hline
\end{tabular}

\section{TABLA 13}

EFECTO OBSERVADO EN NÚMERO DE EMPLEADOS POR TIPO DE EMPRESA SEGÚN EL TIEMPO TRANSCURRIDO DESDE EL INICIO DEL APOYO DE ICEX

\begin{tabular}{|c|c|c|c|c|c|}
\hline \multirow{2}{*}{ Tipo de empresa } & \multicolumn{5}{|c|}{ Desfase desde inicio del apoyo (años) } \\
\hline & 0 & 1 & 2 & 3 & 4 \\
\hline Todas & 0,35 & 0,62 & 0,33 & 0,18 & 0,04 \\
\hline $\begin{array}{l}\text { Microempresas productivas de más de } 10 \text { años de vida y con tímida presencia en el } \\
\text { exterior }\end{array}$ & $-0,03$ & 0,21 & 0,09 & 0,06 & 0,007 \\
\hline $\begin{array}{l}\text { Microempresas poco productivas de menos de } 10 \text { años de vida y tímida presencia en el } \\
\text { exterior }\end{array}$ & $-0,05$ & 0,27 & 0,16 & 0,15 & 0,07 \\
\hline $\begin{array}{l}\text { Empresas medianas y grandes de más de } 15 \text { años de vida muy productivas y volcadas } \\
\text { en el exterior }\end{array}$ & $-0,06$ & 0,15 & 0,08 & 0,04 & 0,01 \\
\hline Microempresas de reciente creación muy productivas y volcadas en el exterior & 0,39 & 0,90 & 0,66 & 0,66 & 0,43 \\
\hline $\begin{array}{l}\text { Microempresas de más de } 10 \text { años de vida muy productivas y muy volcadas en el } \\
\text { exterior }\end{array}$ & 0,33 & 0,65 & 0,47 & 0,39 & 0,31 \\
\hline Pequeñas empresas de más de 12 años de vida volcadas en el exterior & $-0,09$ & $-0,15$ & 0,10 & 0,10 & 0,006 \\
\hline $\begin{array}{l}\text { Microempresas de menos de } 10 \text { años de vida con baja productividad y tímida presencia } \\
\text { en el exterior }\end{array}$ & $-0,31$ & 0,05 & 0,08 & $-0,23$ & $-0,33$ \\
\hline \multicolumn{6}{|c|}{$\begin{array}{l}\text { En desfase } 0 \text { se encuentran tanto las empresas que no han recibido apoyo de ICEX en el periodo como las que recibieron apoyo por primera vez en } 2018 \text {. } \\
\text { Colores distintos muestran valores que son significativamente distintos entre sí. Los tonos verdes indican valores significativamente mayores que } 0 \text { y los } \\
\text { tonos rojos, valores significativamente menores que } 0 \text {. } \\
\text { Los coeficientes implican incrementos porcentuales del número de empleados entre } 2014 \text { y } 2018 \text {. }\end{array}$} \\
\hline \multicolumn{6}{|c|}{ Fuente: CEEPE y elaboración propia. } \\
\hline
\end{tabular}




\section{EL IMPACTO A CORTO Y MEDIO PLAZO DEL APOYO DE ICEX}

TABLA 14

EFECTO OBSERVADO EN PRODUCTIVIDAD (FACTURACIÓN POR EMPLEADO) POR TIPO DE EMPRESA SEGÚN EL TIEMPO TRANSCURRIDO DESDE EL INICIO DEL APOYO DE ICEX

\begin{tabular}{|c|c|c|c|c|c|}
\hline \multirow{2}{*}{ Tipo de empresa } & \multicolumn{5}{|c|}{ Desfase desde inicio del apoyo (años) } \\
\hline & 0 & 1 & 2 & 3 & 4 \\
\hline Todas & 2,69 & 3,23 & 1,61 & 0,84 & 0,17 \\
\hline $\begin{array}{l}\text { Microempresas productivas de más de } 10 \text { años de vida y con tímida presencia en el } \\
\text { exterior }\end{array}$ & $-1,03$ & $-0,53$ & $-0,50$ & $-0,77$ & $-0,87$ \\
\hline $\begin{array}{l}\text { Microempresas poco productivas de menos de } 10 \text { años de vida y tímida presencia en el } \\
\text { exterior }\end{array}$ & 8,25 & 8,64 & 8,50 & 10,09 & 9,39 \\
\hline $\begin{array}{l}\text { Empresas medianas y grandes de más de } 15 \text { años de vida muy productivas y volcadas } \\
\text { en el exterior }\end{array}$ & $-0,33$ & 0,05 & $-0,06$ & $-0,13$ & $-0,14$ \\
\hline Microempresas de reciente creación muy productivas y volcadas en el exterior & 2,52 & 5,02 & 4,21 & 3,48 & 1,98 \\
\hline $\begin{array}{l}\text { Microempresas de más de } 10 \text { años de vida muy productivas y muy volcadas en el } \\
\text { exterior }\end{array}$ & 1,41 & 0,73 & 0,39 & 0,19 & $-0,30$ \\
\hline Pequeñas empresas de más de 12 años de vida volcadas en el exterior & 9,23 & 10,90 & 11,10 & 10,88 & 10,51 \\
\hline $\begin{array}{l}\text { Microempresas de menos de } 10 \text { años de vida con baja productividad y tímida presencia } \\
\text { en el exterior }\end{array}$ & $-1,54$ & 0,83 & 0,08 & 0,00 & $-1,05$ \\
\hline \multicolumn{6}{|c|}{$\begin{array}{l}\text { En desfase } 0 \text { se encuentran tanto las empresas que no han recibido apoyo de ICEX en el periodo como las que recibieron apoyo por primera vez en } 2018 \text {. } \\
\text { Colores distintos muestran valores que son significativamente distintos entre sí. Los tonos verdes indican valores significativamente mayores que } 0 \text { y los } \\
\text { tonos rojos, valores significativamente menores que } 0 \text {. } \\
\text { Los coeficientes implican incrementos porcentuales de productividad entre } 2014 \text { y } 2018 \text {. }\end{array}$} \\
\hline Fuente: CEEPE y elaboración propia. & & & & & \\
\hline
\end{tabular}

TABLA 15

EFECTO OBSERVADO EN INTENSIDAD EXPORTADORA POR TIPO DE EMPRESA SEGÚN EL TIEMPO TRANSCURRIDO DESDE EL INICIO DEL APOYO DE ICEX

\begin{tabular}{|c|c|c|c|c|c|}
\hline \multirow{2}{*}{ Tipo de empresa } & \multicolumn{5}{|c|}{ Desfase desde inicio del apoyo (años) } \\
\hline & 0 & 1 & 2 & 3 & 4 \\
\hline Todas & 0,00 & 0,02 & 0,014 & 0,00 & 0,00 \\
\hline $\begin{array}{l}\text { Microempresas productivas de más de } 10 \text { años de vida y con tímida presencia en el } \\
\text { exterior }\end{array}$ & 0,00 & 0,009 & 0,004 & $-0,005$ & $-0,10$ \\
\hline $\begin{array}{l}\text { Microempresas poco productivas de menos de } 10 \text { años de vida y tímida presencia en el } \\
\text { exterior }\end{array}$ & 0,00 & 0,12 & 0,04 & 0,13 & 0,08 \\
\hline $\begin{array}{l}\text { Empresas medianas y grandes de más de } 15 \text { años de vida muy productivas y volcadas } \\
\text { en el exterior }\end{array}$ & 0,00 & 0,00 & 0,00 & 0,00 & $-0,01$ \\
\hline Microempresas de reciente creación muy productivas y volcadas en el exterior & 0,00 & 0,03 & 0,02 & 0,00 & 0,00 \\
\hline $\begin{array}{l}\text { Microempresas de más de } 10 \text { años de vida muy productivas y muy volcadas en el } \\
\text { exterior }\end{array}$ & 0,00 & 0,00 & 0,01 & 0,00 & 0,00 \\
\hline Pequeñas empresas de más de 12 años de vida volcadas en el exterior & 0,00 & 0,08 & 0,06 & 0,09 & 0,07 \\
\hline $\begin{array}{l}\text { Microempresas de menos de } 10 \text { años de vida con baja productividad y tímida presencia } \\
\text { en el exterior }\end{array}$ & 0,00 & 0,00 & $-0,01$ & 0,00 & $-0,02$ \\
\hline
\end{tabular}

En desfase 0 se encuentran tanto las empresas que no han recibido apoyo de ICEX en el periodo como las que recibieron apoyo por primera vez en 2018. Colores distintos muestran valores que son significativamente distintos entre sí. Los tonos verdes indican valores significativamente mayores que 0 y los tonos rojos, valores significativamente menores que 0 .

Los coeficientes implican incrementos porcentuales de intensidad exportadora entre 2014 y 2018.

Fuente: CEEPE y elaboración propia. 
Los resultados de estas tablas permiten extraer las siguientes conclusiones:

- Nuestro apoyo impulsa las cifras de exportación de bienes en el corto plazo, si bien este crecimiento no siempre se mantiene de forma sostenida en el tiempo.

- En términos de cifra de negocios, aunque no parece haber un patrón común, puede afirmarse que tras un año del apoyo hay un incremento de esta que puede mantenerse o diluirse, a medida que se aleja en el tiempo el inicio del apoyo, y en algunos casos llega a convertirse en un decrecimiento de la cifra de negocios.

- Con respecto al empleo, hay un patrón bastante común que consiste en que, en el primer año tras el apoyo hay un cierto incremento del número de empleados, mayor en microempresas volcadas en el exterior, que se mantiene el segundo año tras el apoyo y se diluye a partir de entonces. Para las pequeñas empresas de más de doce años de vida volcadas en el exterior no hay cambio observable en la plantilla a partir del apoyo, y para las microempresas de menos de diez años de vida con baja productividad y tímida presencia en el exterior se observa un muy ligero incremento de su plantilla, que se pierde completamente a partir del tercer año tras el apoyo.

- De forma general, un año después del apoyo hay una mejora en la productividad de todos los tipos de empresa, no obstante, aquella no es necesariamente sostenida en el tiempo. Es de destacar el hecho de que las microempresas de más de diez años de vida muy productivas y muy volcadas en el exterior que han recibido apoyo tienen peores valores en la mejora de la productividad que las que no han tenido apoyo, lo que, teniendo en cuenta la evidencia mostrada en el Gráfico 4, indica que han alcanzado un grado alto de productividad que no es fácil superar.

- Con carácter general, tras un año del apoyo hay una mejora en la intensidad exportadora (equivalente por la fórmula de cálculo a la cuota de exportación), salvo en las microempresas volcadas en el exterior, entre las que no parece haber variación, lo que en conjunción con la evidencia de los Gráficos 2 y 5 indica que para estas empresas la cuota de exportación se mantiene estable a lo largo del tiempo.

Este análisis exploratorio no distingue los efectos observados según el hecho de que la empresa haya recibido apoyo de ICEX con anterioridad a 2014 o no. Por ello, hemos llevado a cabo también un análisis de comparación múltiple de efectos medios teniendo en cuenta la interacción entre el desfase y el hecho de haber recibido apoyo anteriormente o no (veteranía), separado para cada tipo de empresa. En particular, se ha utilizado un modelo anidado que busca, dentro de cada valor de veteranía, diferencias según el tiempo transcurrido desde el inicio del apoyo. Los resultados para cada una de las variables en estudio se muestran en los Gráficos 7 a 11. En esos gráficos se resume la evolución mostrada en las Tablas 11 a 15 en la parte de «todas las empresas», y se muestra de forma comparada la evolución para aquellas empresas que han recibido apoyo antes de 2014 (veteranas) y las que no lo han recibido (no veteranas), si bien las diferencias mostradas pueden no ser significativas. 
En el Gráfico 7 se ve que la evolución de la cifra de exportación de bienes de las empresas veteranas es similar a la de las no veteranas y que, salvo para el clúster 7 (microempresas de menos de diez años de vida con baja productividad y tímida presencia en el exterior), las no veteranas incrementan sus cifras de exportación de bienes tras el apoyo, mientras que las veteranas vuelven a valores previos de exportación tras el apoyo, pero no consiguen mantenerlos. Esto puede indicar que estas últimas recurren al apoyo de ICEX tras observar un descenso apreciable en sus cifras de exportación de bienes, consiguen remontarlas puntualmente, pero no de forma sostenida.

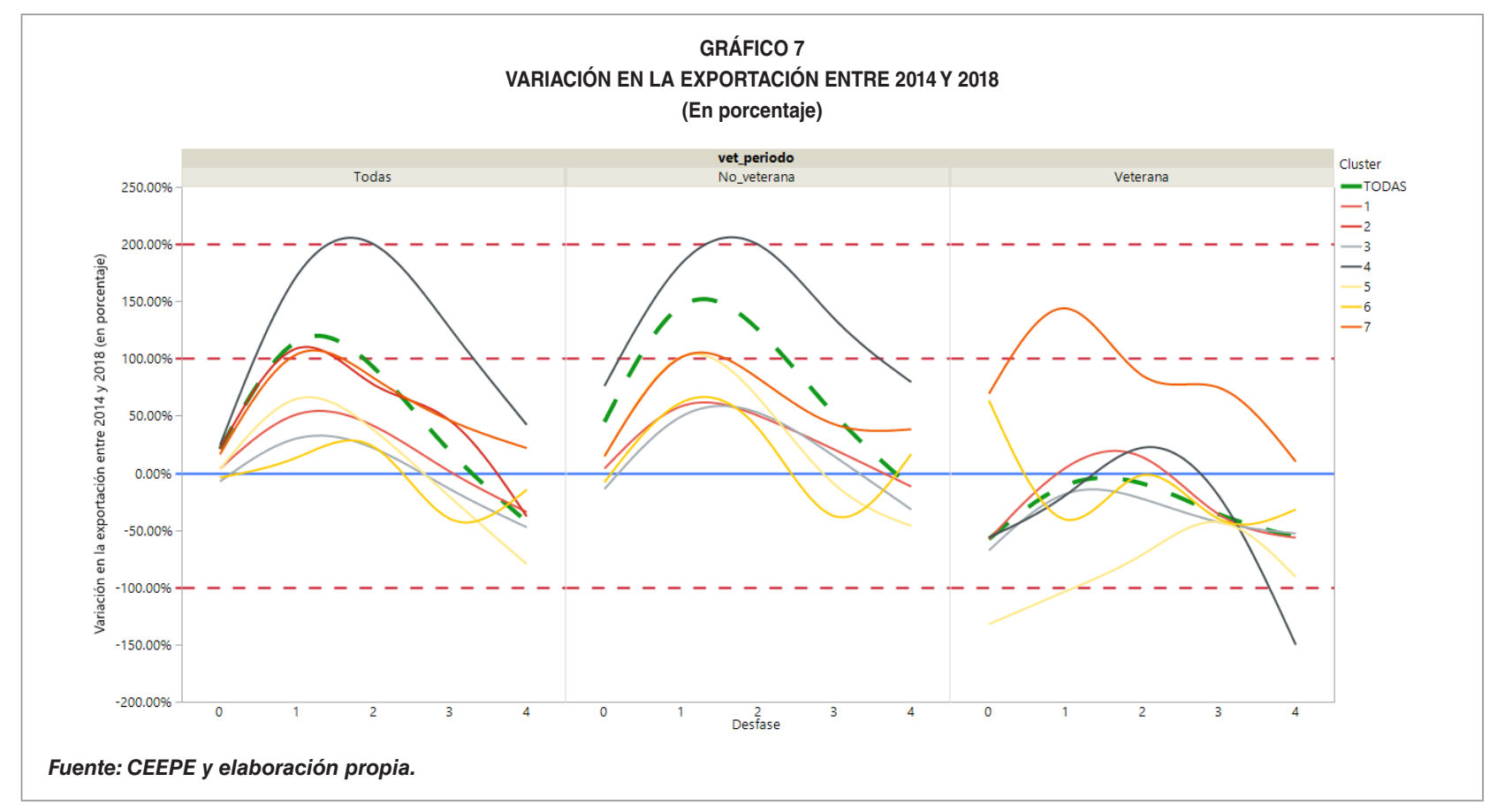

GRÁFICO 8

EFECTO OBSERVADO EN CIFRA DE NEGOCIOS POR ESTADO DE VETERANÍA SEGÚN EL TIEMPO TRANSCURRIDO DESDE EL INICIO DEL APOYO DE ICEX

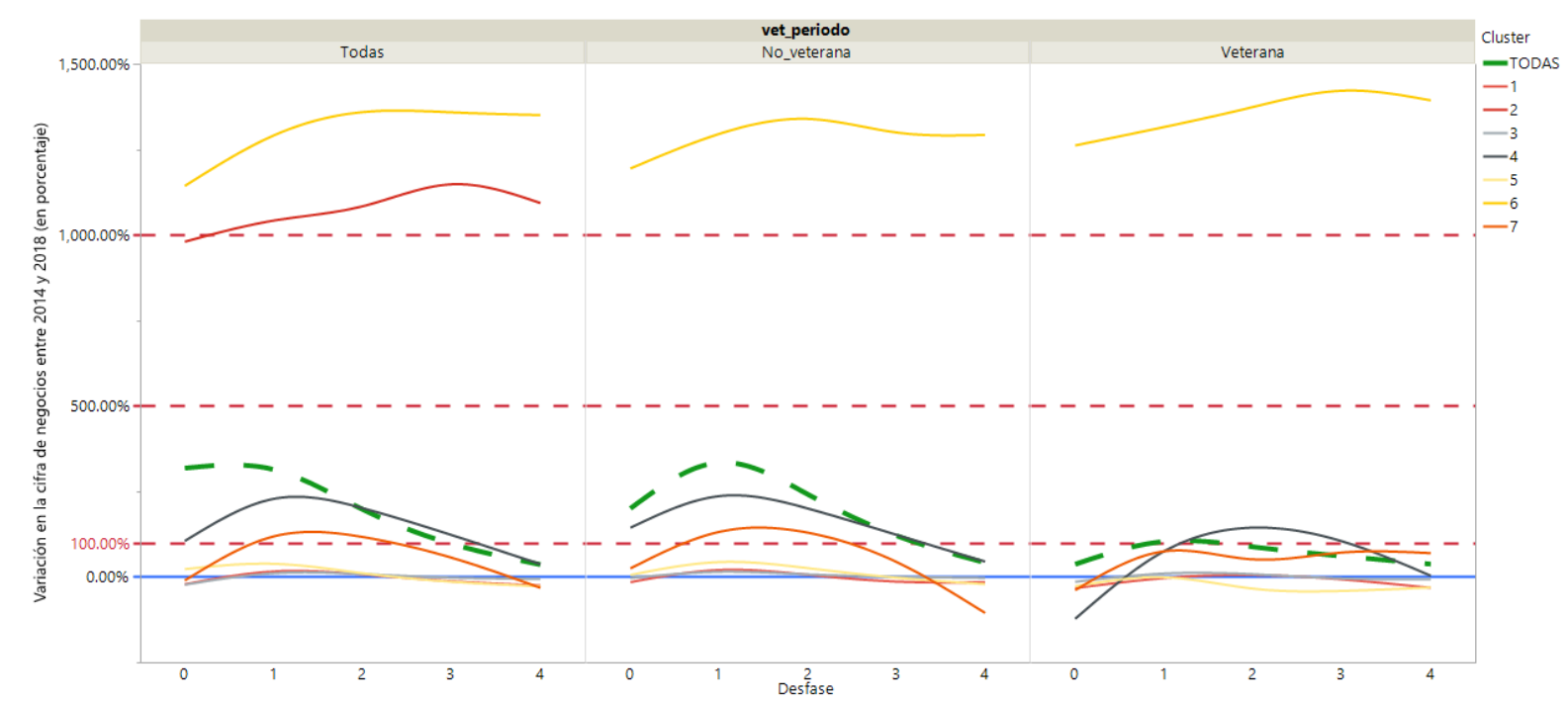

Fuente: CEEPE y elaboración propia. 
En el Gráfico 8 se ve que la evolución de la cifra de negocios de las empresas veteranas es similar a la de las no veteranas y que para los clústeres 2 y 6 (microempresas poco productivas de menos de diez años de vida y tímida presencia en el exterior y pequeñas empresas de más de doce años de vida volcadas en el exterior) los incrementos en la cifra de negocios son muy abultados, pues llegan a multiplicar por 10 sus cifras iniciales.

En el Gráfico 9 se ve que la evolución de la plantilla de las empresas veteranas es similar a la de las no veteranas, salvo para el clúster 4 (microempresas de reciente creación muy productivas y volcadas en el exterior), en el que las empresas veteranas mantienen estable su plantilla tras el apoyo, mientras que las no veteranas experimentan un notable incremento en los primeros dos años que se amortigua a partir de entonces.

En el Gráfico 10 se ve que la evolución de la intensidad exportadora resulta en general más caótica en las empresas que han recibido apoyo de ICEX de forma previa, que pierden cuota cuatro años después del apoyo. Las empresas no veteranas logran mantener su cuota de forma general. Cabe mencionar, como caso especial, que las empresas de los clústeres 2 y 6 (microempresas poco productivas de menos de diez años de vida y tímida presencia en el exterior y pequeñas empresas de más de doce años de vida volcadas en el exterior) experimentan crecimientos de entre el $5 \%$ y el $10 \%$ de la cuota, que, salvo las veteranas, logran mantener incluso tras cuatro años inicio del apoyo.

En el Gráfico 11 se ve que la evolución de la productividad es similar para el caso de las veteranas y las no veteranas. De nuevo, es destacable que las empresas de los clústeres 2 y 6 (microempresas poco productivas de menos de diez años de vida y tímida presencia en el exterior y pequeñas empresas de más de doce años de vida volcadas en el exterior) experimentan crecimientos adicionales en su productividad tras el apoyo, que, salvo las veteranas, logran mantener incluso tras cuatro años del inicio del apoyo. $\quad \square$

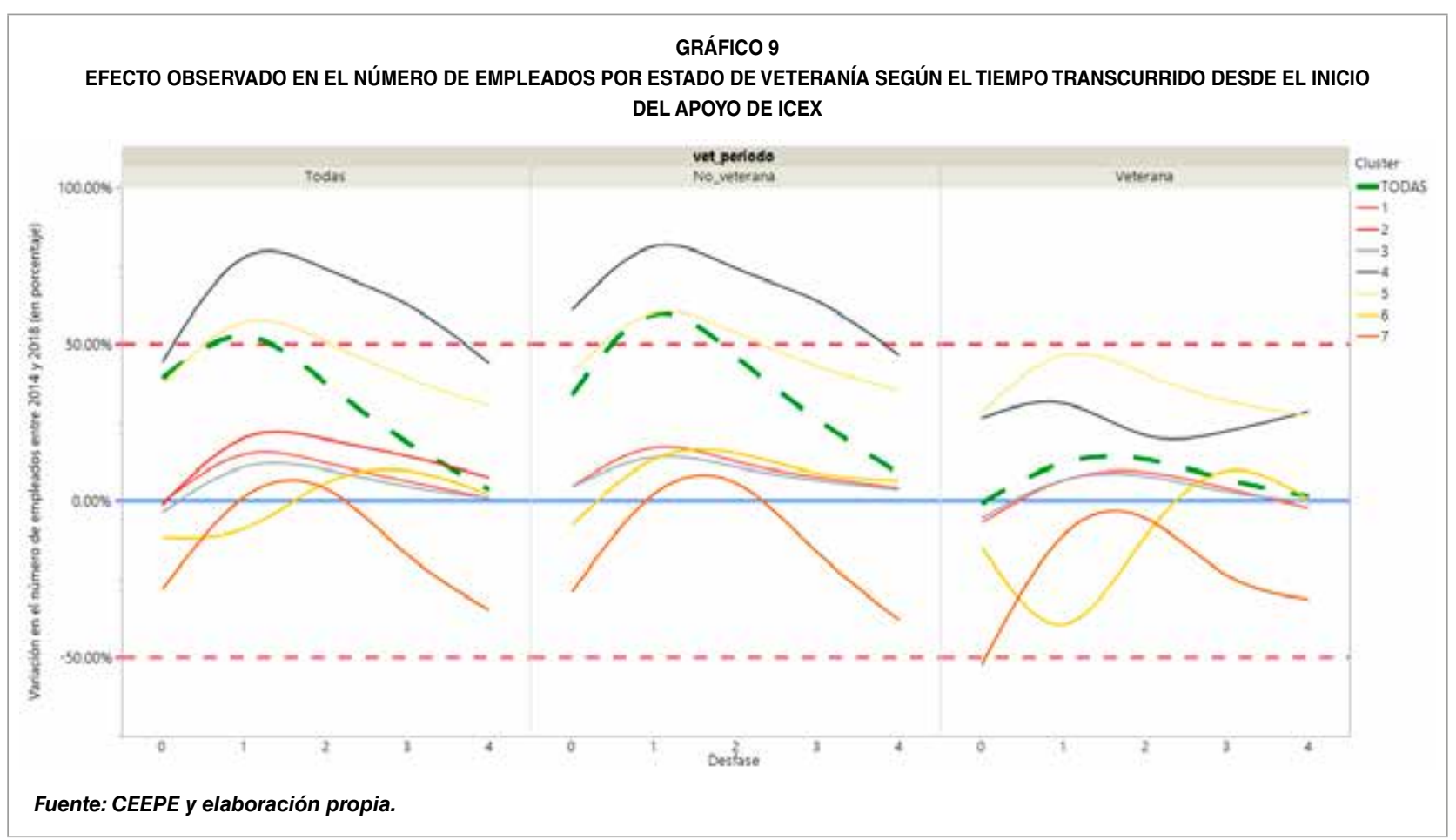



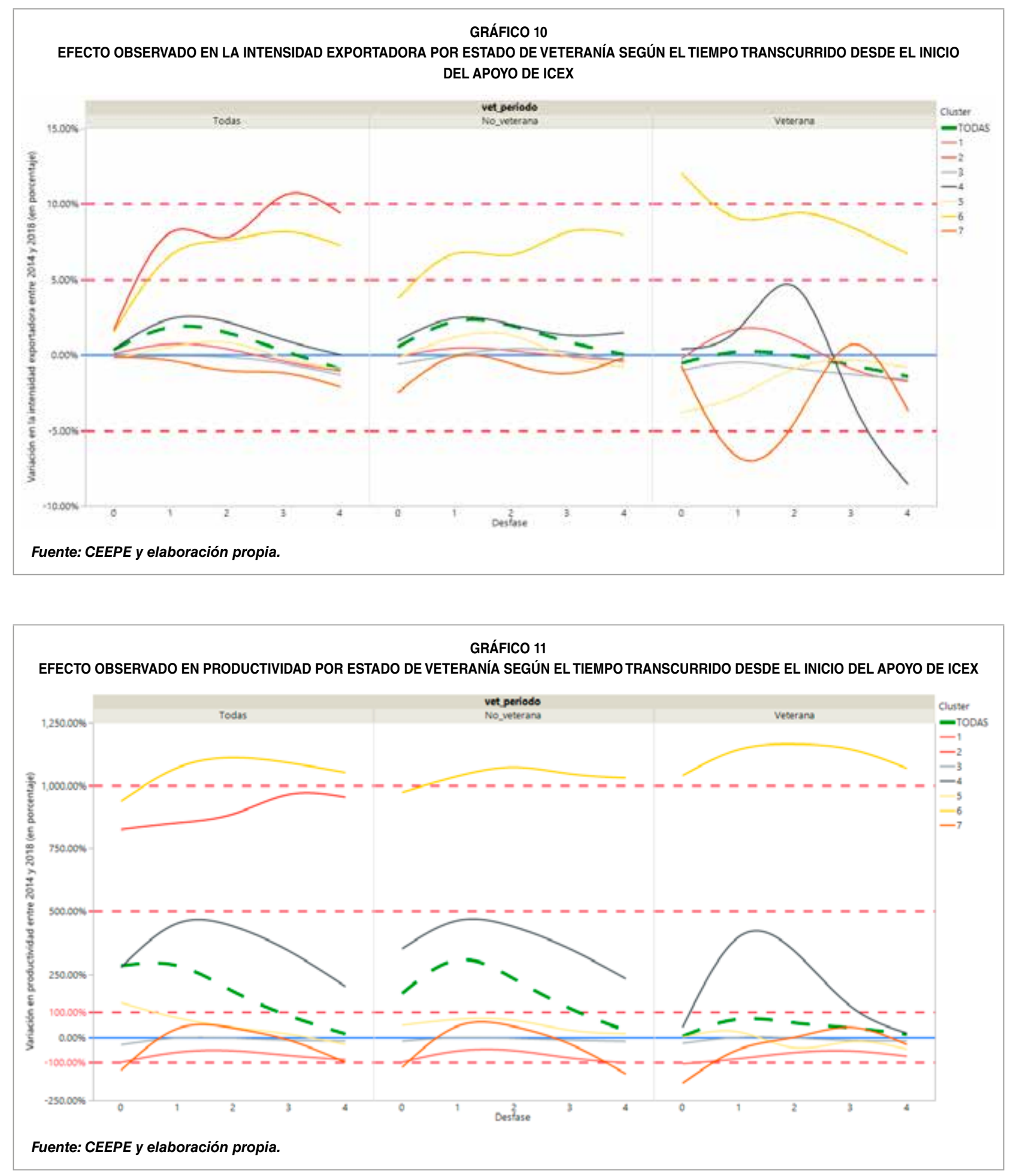

\section{Principales conclusiones}

De todo este ejercicio se han extraído conclusiones generales que consideramos útiles para la toma de decisiones y que se recogen a continuación de forma diferenciada por tipo de empresa.

En general, las empresas que han recibido apoyo de ICEX en el periodo 2014-2018 muestran cifras más elevadas en términos de $D$ 
exportación de bienes, cifra de negocios, empleados, productividad e intensidad exportadora que las que no han recibido estos apoyos, lo cual puede indicar un sesgo de selección del colectivo de empresas que acuden a la institución.

Tras el apoyo de ICEX, se observa un incremento en la cifra de negocios y la exportación de bienes. Para aquellas empresas que no han recibido apoyo de ICEX, el crecimiento de la cifra de negocios es cercano al $300 \%$, cuestión que enmascara que el grueso de estas empresas lo constituyen empresas de reciente creación que suelen tener un crecimiento en ventas inicial en el mercado nacional muy grande. Sin embargo, la mayoría de las empresas que reciben apoyo de ICEX no suelen ser empresas de reciente creación y, cuando estas se plantean un proceso de internacionalización, cuentan con una red de ventas nacional estable.

Las empresas que no han tenido apoyo previamente de ICEX recurren a este cuando sus cifras de exportación han experimentado un aumento, y tras el apoyo se observa un crecimiento muy acusado tanto de su cifra de negocios como de su exportación, que decae pasados cuatro años del inicio del apoyo.

Las empresas que han tenido apoyo previamente vuelven a recurrir a ICEX cuando ven una sustancial caída en sus ingresos por exportación (tanto en el volumen como en la cuota), y tras uno o dos años del nuevo apoyo logran mantener el volumen anterior, pero este vuelve a caer cuatro años después del inicio del apoyo.

La caída de la cifra de exportación pasados cuatro años del inicio del apoyo, en algunos casos, puede enmascarar el hecho de que algunas empresas dejan de exportar desde España, pues se implantan productivamente en el exterior o son adquiridas por un grupo empresarial.
En general, el apoyo de ICEX supone un incremento en las cifras de exportación de bienes cuantificable, en el peor de los casos, en un tercio debido al aumento de la productividad en el periodo. Por su parte, el apoyo de ICEX supone también un incremento en el número de empleados que, en el peor de los casos, no compensa del todo la disminución de empleados debido al incremento de la productividad.

Por tipo de empresa, podemos ofrecer las siguientes conclusiones particularizadas.

Microempresas productivas de más de diez años de vida y con tímida presencia en el exterior. Para las empresas no veteranas, el apoyo de ICEX contribuye a incrementar la cifra de exportación de bienes, cifra de negocios, número de empleados y productividad. Para las veteranas, se atribuye al apoyo de ICEX solo parte del incremento en número de empleados y productividad. Sin embargo, este apoyo por sí solo no parece ser suficiente para que se mantengan en el mercado internacional.

Microempresas poco productivas de menos de diez años de vida y tímida presencia en el exterior. Estas empresas experimentan incrementos en todas las variables de interés, aun cuando no cabe atribuir ninguna parte de estos incrementos al apoyo de ICEX, lo que implica un sesgo de selección: es decir, acuden a ICEX aquellas empresas con una inercia previa en todas estas magnitudes y saben aprovechar el apoyo prestado.

Empresas medianas y grandes de más de quince años de vida muy productivas y volcadas en el exterior. Para las empresas no veteranas, el apoyo de ICEX contribuye a aumentar todas las magnitudes de interés. Para las veteranas, el apoyo de ICEX provoca una disminución de la exportación de bienes, pero hay atribución al crecimiento tanto de la cifra de $\triangleright$ 
negocios como del número de empleados, lo que, teniendo en cuenta los resultados esperables del apoyo que no podemos capturar con los datos disponibles de acuerdo con la lógica de intervención reconstruida, implica que estas empresas pueden haber abierto líneas de exportación de servicios o haber realizado implantaciones productivas en el exterior, y que parte de esta implantación se puede deber a sus buenos resultados gracias al apoyo previo de ICEX. Al no haber variación en la intensidad exportadora tras el apoyo, el crecimiento de estas empresas está equilibrado entre el mercado internacional y el mercado nacional, lo que puede indicar que, en la estrategia de estas empresas, no hay diferencia intrínseca entre ambos.

Microempresas de reciente creación muy productivas y volcadas en el exterior. Para las empresas no veteranas el apoyo de ICEX contribuye al gran incremento que experimentan en exportación de bienes, cifra de negocios, número de empleados y productividad, mientras que para las veteranas no hay evidencia de tal atribución de ningún efecto al apoyo de ICEX. La buena marcha de todas estas magnitudes a partir del inicio del apoyo, se haya o no recibido apoyo previo por parte de ICEX, implica que el apoyo del instituto contribuye notablemente a que estas empresas levanten el vuelo muy rápidamente, y tras el primer o segundo año después del primer apoyo estas son capaces de seguir creciendo en todas las magnitudes por sí mismas.

Microempresas de más de diez años de vida muy productivas y muy volcadas en el exterior. Para las empresas no veteranas el apoyo de ICEX contribuye a aumentar la cifra de negocios, el número de empleados y la productividad, pero no así la cifra de exportación de bienes. Para las veteranas el apoyo de ICEX provoca una disminución de la exportación de bienes, pero hay atribución al crecimiento tanto de la cifra de negocios como del número de empleados y la productividad. Teniendo en cuenta los resultados esperables del apoyo que no podemos capturar con los datos disponibles de acuerdo con la lógica de intervención reconstruida así como el parón en su productividad a medio plazo, que se materializa en el estancamiento en su crecimiento exterior cuatro años tras el apoyo, parece claro que, para consolidar su estrategia internacional, estas empresas requieren más apoyo que el inicial de ICEX y en ámbitos que no hemos explorado hasta ahora o donde no tenemos competencias, si bien, una vez han pasado este bache (ejemplificado en una parte de las veteranas), pueden haberse implantado de forma productiva en el exterior (sin excluir que parte de su producción haya sido adquirida por alguna empresa local), lo que en sí constituye un buen resultado atribuible en parte al apoyo previo de ICEX. Al no haber variación en la intensidad exportadora tras el apoyo, el crecimiento de estas empresas está equilibrado entre el mercado internacional y el mercado nacional, lo que puede indicar que, en la estrategia de estas empresas, no hay diferencia intrínseca entre ambos.

Pequeñas empresas de más de doce años de vida volcadas en el exterior. Para las empresas no veteranas el apoyo de ICEX contribuye al incremento en la cifra de negocios, el número de empleados y la productividad. Para las veteranas se atribuye al apoyo de ICEX el incremento en la cifra de negocios y la productividad. Para estas empresas, el apoyo de ICEX, por tanto, contribuye a que puedan tener las plantillas necesarias y a mantenerlas con posterioridad, y apoya igualmente la buena marcha de las empresas en todas sus $\square$ 
magnitudes. Igual que en algún caso anterior, esta buena marcha en facturación sin que haya un incremento en la exportación de bienes puede implicar bien la existencia de líneas de exportación de servicios, bien la implantación productiva en el exterior, lo que es un resultado también atribuible en parte al apoyo previo de ICEX.

Microempresas de menos de diez años de vida con baja productividad y tímida presencia en el exterior. Para las empresas no veteranas el apoyo de ICEX contribuye al incremento en exportación de bienes, cifra de negocios y productividad. Para las veteranas el apoyo de ICEX contribuye al incremento en exportación de bienes y número de empleados. Teniendo en cuenta la evolución de las magnitudes a partir del apoyo, el ICEX contribuye a una rápida entrada en el mercado exterior, acompañado de un crecimiento en el mercado nacional que, sin embargo, no termina de consolidarse. De acuerdo con los riesgos identificados en la lógica de intervención reconstruida, estos resultados parecen indicar una eventual falta de solvencia y de competitividad inicial de estas empresas, y estaríamos hablando, por tanto, de empresas cuya actividad exterior está más ligada con pedidos esporádicos que con un control sobre su estrategia comercial.

\section{Principales recomendaciones}

Las conclusiones extraídas entendemos que pueden y deben ser contextualizadas en una situación de gran complejidad e incertidumbre a la que todas las empresas se están enfrentando, en un entorno sin precedentes sujeto a profundos cambios y retos, que exige una reacción ágil de las instituciones para entender las dificultades y problemáticas que se están afrontando con el objetivo de diseñar y proporcionar apoyos eficaces y soluciones flexibles y versátiles que favorezcan la toma de decisiones empresariales estratégicas que aseguren su continuidad e impulso internacional y fortalezcan su resiliencia.

En este sentido, el estudio realizado y los resultados alcanzados permiten formular las siguientes recomendaciones:

Ampliar el número de empresas con potencial de internacionalización a las que poder ofrecer servicio atendiendo a sus características y necesidades, partiendo de los perfiles y tipologías ya definidas y priorizando los grupos en función de su perfil y de la disponibilidad de los apoyos.

Profundizar en el análisis de los tipos de apoyo prestados por ICEX a las empresas, pues si bien se observa que los efectos atribuibles a este apoyo son positivos en la evolución de todas las variables estudiadas -aunque con un impacto desigual según los grupos de empresas-, dado el amplio abanico de instrumentos disponible, resulta relevante poder trazar el impacto diferencial en las magnitudes analizadas, tanto a corto como a medio plazo, por tipologías de apoyo y grupos de empresas. Esto nos permitirá poder definir y proponer los apoyos óptimos y más efectivos según los perfiles.

Hacer un seguimiento de la empresa apoyada para poder ofrecerle un acompañamiento efectivo y continuado a través de servicios adicionales que anticipen sus necesidades presentes y futuras, de modo que el impulso que provea el apoyo de ICEX al principio pueda ser sostenido a medio y largo plazo. Esta labor de acompañamiento debe ser continua, personalizada e integral, superando visiones parciales, de forma que se ofrezca y proporcione el $\triangleright$ 
instrumento o el servicio que, en cada fase del proceso de internacionalización, la empresa requiera según sus características y necesidades, en términos de resultados y de mejora de las competencias clave. En este sentido, una gestión del cliente de ICEX más global contribuiría a maximizar el resultado del uso de los recursos públicos y a consolidar en el tiempo los efectos positivos del apoyo recibido.

Estas recomendaciones, que contemplan atender un mayor número de empresas, seleccionadas en función de su perfil y potencial de internacionalización, y evolucionar a un tipo de acompañamiento con un enfoque más transversal, próximo y continuado, basado en el perfil específico y las necesidades de cada empresa y en la efectividad del apoyo en términos de resultados, están perfectamente alineadas con varios de los nuevos proyectos de ICEX, que facilitarán también la explotación interna del conocimiento de los clientes y la identificación de los servicios más adecuados para ellos, así como los argumentos explicativos y las ventajas esperables, basado en un modelo de autoaprendizaje que irá perfeccionándose progresivamente. El valor añadido que aportará supondrá, sin duda, un importante impulso al enfoque al cliente que el proyecto CRM Global ICEX viene consolidando en la organización y contribuirá decisivamente a la explotación de la analítica de datos como palanca de gestión interna y de toma de decisiones en el marco del proceso de transformación digital en el que nos hallamos inmersos.

De forma complementaria, la red de Oficinas Económicas y Comerciales continuará siendo actor clave en la atención y asesoramiento especializado a las empresas al disponer, en tiempo real, de valiosa información de los mercados que puede convertirse en oportunidades de negocio. Su proximidad y conocimiento de los cambios que se están produciendo resulta esencial para las empresas a la hora de identificar y minimizar riesgos, promoviendo la toma de decisiones basada en la inteligencia competitiva que pueda conducirlas a una mejor diversificación y estabilidad en los mercados internacionales.

Los resultados de las evaluaciones de necesidades realizadas y en curso proporcionan, adicionalmente, una información clave en estos momentos para conocer con mayor precisión los aspectos y áreas en los que las empresas demandan una mayor atención y apoyo en esta situación tan disruptiva y volátil, con nuevos retos y riesgos y con un impacto asimétrico tanto sectorial como geográfico.

La flexibilización de servicios ya existentes, el enriquecimiento de algunos instrumentos o el desarrollo de nuevas herramientas se configuran como mecanismos eficaces para impulsar y mantener la presencia internacional de las empresas y para consolidar los efectos positivos atribuidos a nuestro apoyo, si bien su implementación conlleva retos internos que en algunos casos ya están siendo abordados pero que deberán reforzarse y afianzarse. La apuesta por las nuevas tecnologías como aliadas imprescindibles para la toma de decisiones basadas en los datos, el análisis y la anticipación requerirá de mayores recursos en ICEX, tanto en términos financieros como de perfiles especializados que en estos momentos escasean en la plantilla.

Finalmente, son claras y muy destacables las ventajas de la colaboración institucional y con el ámbito académico que permiten el análisis de los datos provenientes de fuentes de distintos organismos y entidades, ya que proporcionan un alcance mucho más potente y unos resultados y conclusiones de gran riqueza y utilidad, como muestra este estudio. En $\triangleright$ 
Joaquín María Núñez Varo y María del Mar Gómez González

consecuencia, se considera conveniente fortalecer las líneas de trabajo orientadas a intensificar las evaluaciones y a profundizar en los análisis transversales que ayuden a obtener un mayor conocimiento de las intervenciones más efectivas por tipos de empresa y de las variables clave en términos de resultados para que el acompañamiento de ICEX proporcione unos resultados más exitosos y duraderos en los itinerarios internacionales de las empresas.

\section{Bibliografía}

Ariño, M.A. (2017). Evolución del tejido empresarial español de 2006 a 2014. (WP-1179) IESE Business School. https://media.iese.edu/research/ WP-1179.pdf

Gertler, P. J., Martinez, S., Premand, P., Rawlings, L. B., Vermeersch, C. M. J. (2016). Impact EvaIuation in Practice, Second Edition. Inter-American Development Bank and World Bank. https:// openknowledge.worldbank.org/handle/10986/ 25030

Helpman, E., Melitz, M.J., y Yeaple, S.R. (2003). Export versus FDI. NBER Working Paper Series, (9439). National Bureau of Economic Research. https://www.nber.org./papers/w9439.pdf

Muendler, M.A. (2014). Export or merge? Proximity vs. concentration in product space. NBER Working Paper, (19751). https://wwwnber.org/papers/w19751

Núñez Varo, J.M. (2017). La evaluación del apoyo público a la internacionalización empresarial. Academia.https://www.academia.edu/37555717/
La_evaluaci\%C3\%B3n_del_apoyo_p\%C3 \%BAblico_a_la_internacionalizaci\%C3\%B3n_ empresarial

Nuñez Varo, J., y Gómez González, M. del M. (2020). El valor estratégico de la evaluación en ICEX. Boletín Económico de ICE (3123). https://doi. org/10.32796/bice.2020.3123.7003

Pérez López, C. y Moral Arce, I. (2015). Técnicas de evaluación de impacto. Garceta.

Prados de la Escosura, L. (2017). Spanish Economic Growth, 1850-2015. Palgrave Macmillan. https://doi.org/10.1007/978-3-319-58042-5

Subdirección General de Estrategia de Internacionalización (2019). El Plan de Acción para la Internacionalización de la Economía Española 2019-2020. Boletín Económico de ICE (3111). https://doi.org/10.32796/bice.2019.3111.6827

Subdirección General de Estudios y Evaluación de Instrumentos de Política Comercial (2020). Informe de Evaluación del Plan de Acción para la Internacionalización de la Economía Española 2017-2018. https://comercio.gob.es/es-es/estrategia_internacionalizacion/Paginas/informe-evaluacion-plan-2017_18.aspx

UKTI (5 june 2014). Technical Note: UKTI's monitoring and evaluation evidence. https://www. gov.uk/government/publications/technicalnote-uktis-monitoring-and-evaluation-evidence/ technical-note-uktis-monitoring-and-evaluation-evidence

Yang-Ming, C., y Gayle, P.G. (2009). Exports versus FDI: do firms use FDI as a mechanism to smooth demand volatility? Review of World Economics. 145 (3), 447-467. https://www.jstor.org/stable/ 40587823 